\title{
Etodolac improves collagen induced rheumatoid arthritis in rats by inhibiting synovial inflammation, fibrosis and hyperplasia
}

Qin Feng ${ }^{1,2,3}$, Wenkai Xia ${ }^{1,2}$, Shenglan Wang ${ }^{1,2}$, Guoxin Dai ${ }^{1,2}$, Weimei Jiao ${ }^{1,2}$, Na Guo $^{1,2}$, Honghua Li ${ }^{1,2}$ and Guimin Zhang ${ }^{1,2,3^{*}}$

\begin{abstract}
Synovial hyperplasia is the main cause of chronic rheumatoid arthritis (RA), but the mechanism of synovial hyperplasia is still unclear. Etodolac (ETD) is a selective COX-2 inhibitor for relieving pain and stiffness in RA, but the disease modifying effect is still lack of evidence. Proteomics method was used to study the differential proteome of synovial tissue in collagen induced arthritis (CIA) in rats. With the help of STRING analysis, the upregulated proteins enriched in the cluster of complement and coagulation cascades and platelet degranulation were highlighted, these proteins with fibrogenic factors Lum, CIV, CXI and Tgfbi participated in the synovial inflammation, fibrosis and hyperplasia in CIA. Based on KOG function class analysis, the proteins involved in the events of the central dogma was explored. They might be hyperplasia related proteins for most of them are related to the proliferation of cancer. ETD significantly attenuated synovial inflammation, fibrosis and hyperplasia in CIA rats by downregulating these proteins. Several proteins have not been observed in RA so far, such as Tmsb4x, Pura, Nfic, Ruvbl1, Snrpd3, U2af2, Srrm2, Srsf7, Elav11, Hnrnph1, Wars, Yars, Bzw2, Mcts1, Eif4b, Ctsh, Lamp1, Dpp7, Ptges3, Cdc37 and Septin9, they might be potentials targets for RA. Blood biochemistry tests showed the safety of 7 months use of ETD on rats. In conclusion, present study displayed a comprehensive mechanism of synovial hyperplasia in CIA rats, on this basis, the clinical value of ETD in the treatment of RA was well confirmed.
\end{abstract}

Keywords: Rheumatoid arthritis, Synovial hyperplasia, Proteomics, Etodolac

\section{Instruction}

RA is a chronic systemic autoimmune disease. It mainly invades joints all over the body, presenting multiple and symmetrical chronic proliferative synovitis, which leads to destruction of articular cartilage and joint capsule, and finally leads to ankylosis of joints [1]. In addition, it seriously affects the health of patients, causing persistent pain and leading to severe disability. The etiology of RA

\footnotetext{
*Correspondence: gmzhanglunan@163.com

'Lunan Pharmaceutical Group Co., Ltd., Linyi, China

${ }^{2}$ Center for New Drug Safety Evaluation of Lunan Pharmaceutical, Lunan

Pharmaceutical Group Co. Ltd., Linyi, China

Full list of author information is available at the end of the article
}

is still unclear. It is generally believed that genetic factors and environmental factors play important roles in the etiology of rheumatoid arthritis.

The key therapeutic goal of RA is remission, no active arthritis, no erosion or deterioration of function. Other important goals are to reduce disease activity and pain, maintain function, and retain work and recreational activities. Currently, glucocorticoids and non-steroidal anti-inflammatory drugs (NSAIDs) were used for the treatment of RA, mainly for the control of pain and inflammation, disease modifying anti rheumatic drugs, as the first-line drugs for the new diagnosis of RA, and biotherapy, which were used to target and inhibit 
specific molecules of immune and inflammatory response [2].

NSAIDs are used widely to control symptoms of RA for lessen pain and stiffness. It is well known that the quality of life in arthritis patients receiving NSAIDs has been improved significantly, but they have lost their historical role as first-line treatment because of concerns about their limited effectiveness, inability to modify the long-term course of disease, and gastrointestinal and cardiac toxic effects [3]. But the risks are different from the types of NSAIDs. And there are studies showing that the effects of NSAIDs are more than control symptoms but also have the effects of alleviating disease and delaying the progress of RA [4]. All above, the use of NSAIDs is still certainly needed, and it is very important to choose safe and effective NSAIDs for long-term use. The ideal NSAIDs should inhibit prostaglandin (PG) synthesis in the inflammatory site, not in the gastric mucosa. Experimental and clinical data support that ETD meets this standard. Long-term daily administration of ETD effective anti-inflammatory dose (rat $3 \mathrm{mg} \cdot \mathrm{kg}^{-1} ; 600 \mathrm{mg}$ in humans) had no effect on PGF2 and prostacyclin in gastric mucosa. The selectivity of ETD to COX-2 was 10 times, and the retention of COX-1 activity in gastric mucosa led to a significant increase in gastric tolerance of ETD [5]. ETD had anti-inflammatory effects with better gastric safety on adjuvant arthritis and normal rats than that of non-selective NSAIDs diclofenac sodium and indomethacin [6]. Compared with other NSAIDs, ETD has shown good efficacy, good gastric tolerance and fewer cardiovascular adverse reactions in various clinical trials, and has minimal hepatic or renal effects $[7,8]$. Furthermore, several studies have shown that ETD has anti-proliferative and anti-metastasis effects of cancer [9, 10], while the synovium in RA also has abnormal proliferation, and RA also has metastasis, so we speculated that the ETD might also inhibit the hyperplasia and metastasis of RA.

Data independent acquisition (DIA) mass spectrometry (MS) is one of the most popular mass spectrometry acquisition technologies in recent years, which once led to the new development of quantitative proteomics [11]. Parallel reaction monitoring (PRM) is the gold standard of targeted proteomics. Because no specific antibody is needed, PRM-MS is expected to replace traditional western blot and other verification methods in the future [12]. DIA and PRM showed comparable linearity, accuracy and precision in differential proteome [13]. Here, DIA-based quantitative proteomics was used to explore the differential proteins in synovium between CIA rats and normal rats, and that between CIA rats and ETD treatment rats, and PRMtargeted proteomics was used to validate the results. Based on omics data, the mechanisms hidden behind the protective effects of ETD on RA were further investigated.

\section{Results}

ETD significantly modified the symptoms of $\mathrm{CIA}$ rats (including gross view, arthritis index, joint swelling degree and pain behavior)

Rats in the control group demonstrated no gross signs of arthritis. Immunization with collagen was associated with expected increases in right heel width, and the swelling degree reached its peak at 30 days after immunization. After that, the swelling degree decreased slightly, but it was still obvious. The secondary swelling appeared around the 10th day after immunization, showing swelling of contralateral foot and forefoot, the total score reached its peak around 2 months after immunization, since then, the right hind feet were significantly swollen and deformed, and the secondary lesions of contralateral foot were serious in vehicle- CIA rats throughout the study. Immunization with collagen was associated with increased pain behavior measured as reduced paw withdrawal thresholds. The pain threshold decreased sharply immediately after immunization, and it elevated slightly with time, but still significantly lower than that of the normal control group.

Two weeks after immunization, ETD was given to rats daily as a single dose by gavage for 7 months. Since half a month after administration ETD, the symptoms were relieved, the right heel width and total score were significantly decreased and the pain threshold was significantly increased, and the symptoms were significantly relieved during the whole 7 months (Fig. 1a, b and c). Seven months after immunization, the swelling and deformation of the feet were mild in the ETD treatment group (Fig. 1d).

\section{ETD significantly alleviated the pathological changes of synovium in CIA rats}

At the end of experiment, the pathological examination of synovial tissues was carried and analyzed. The normal synovial lining is smooth and consists of two to three layers of synovial cells. The proliferative synovial lining showed infiltration of plasma cells, macrophages and lymphocytes as well as proliferative synovial cells. Chronic inflammation leads to neovascularization and fibrous tissue proliferation, which all cause irregular synovial thickening. Masson trichrome staining is a method to identify collagen. Hyperplastic synovial lining showed positive staining of Masson (Fig. 1e). Synovial hyperplasia and inflammatory cells infiltrating were severe in vehicle treated group, and they were significantly alleviated in ETD treatment group (Fig. 1e). Each components score (inflammatory cell infiltration, synovial cells proliferation, neovascularization and fibrous tissue proliferation) and total score total synovitis score (TSS) were significantly increased in vehicle-treated model group, and they were significantly decreased in ETD treatment group (Table 1). 


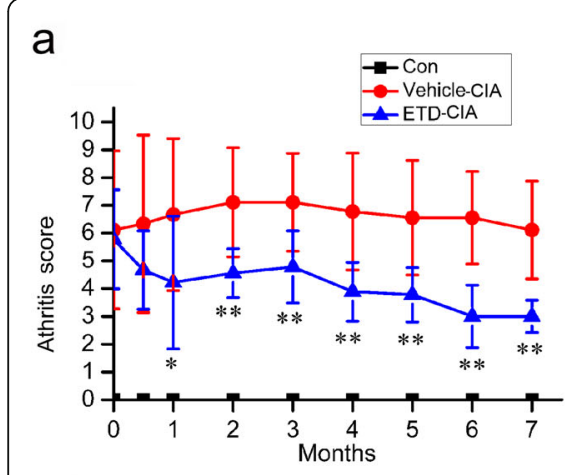

d


b

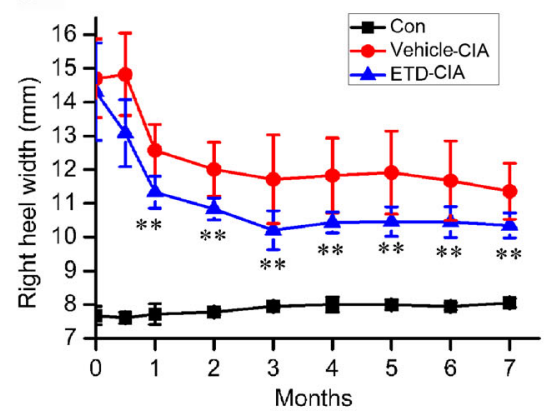

C

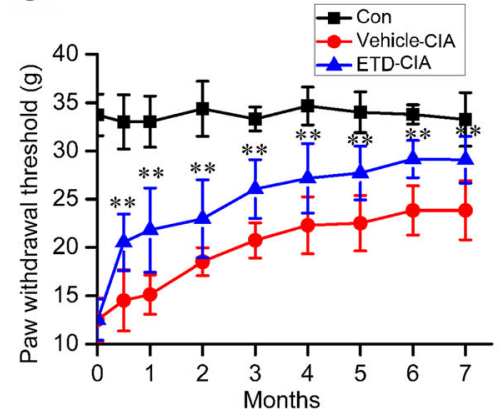

e
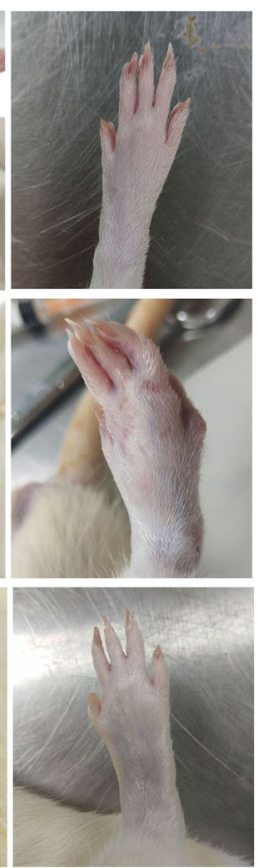

$\mathrm{HE}$

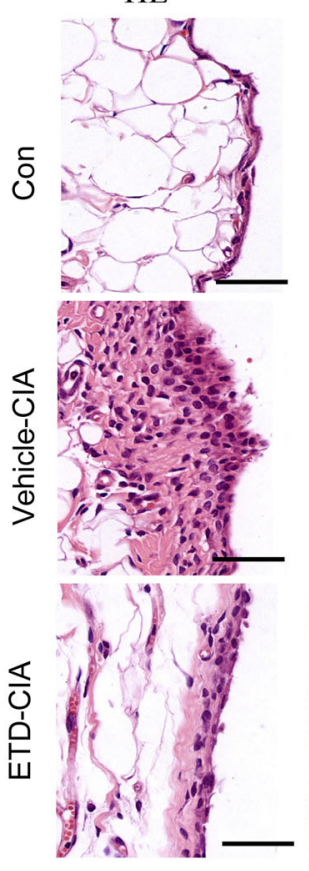

Masson

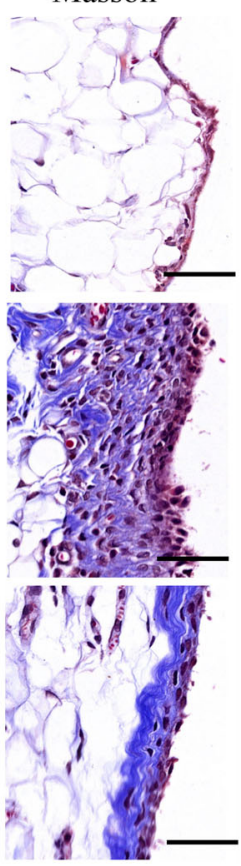

Fig. 1 Effects of ETD on CIA rats. Vehicle-CIA rats had obvious symptoms of arthritis measured as increased total scores (a) and the right heel width (b) through 7 months after collagen injection compared with saline-injected controls, and the paw withdrawal thresholds was significantly decreased (c). The total scores and right heel width were significantly decreased, the paw withdrawal thresholds were significantly increased in ETD-CIA rats, ${ }^{* *} p<0.01$ compared with Vehicle-CIA rats $(n=8)$. $\mathbf{d}$ The appearance of both hind limbs 7 months after modeling, the right hind foot was significantly swollen and deformed, the secondary lesions of contralateral foot were serious in vehicle-treated group, while in ETD-CIA rats, the swelling and deformation of the feet were mild $(n=8)$. e Representative micrographs depicting HE stained and Masson synovial sections observed under $40 \times$ objectives. Scale bars, $50 \mu \mathrm{m}$. The synovial histopathology of control group showed the normal structure of the synovial lining with clear and smooth features, without hyperplasia. The rats in the vehicle-treated model group showed infiltration of plasma cells, macrophages and lymphocytes as well as proliferative synovial cells, and synovial lining showed significant thickening and positive staining of Masson. The lesions in ETD-CIA rats were alleviated significantly. C: Control rats; V: Vehicle-CIA rats; E: ETD-CIA rats

\section{DIA-based proteomics analysis}

A total of 4934 proteins were identified in this study (Supplementary Table S1), among which 2463 proteins were quantitated (Supplementary Table S2). The proteomic analysis highlighted 367 differentially expressed proteins, including 261 upregulated ones and 106 downregulated ones between vehicle-treated group and the control group; 203 differentially expressed proteins between vehicle-treated group and ETD-treated group, among them, 41 were upregulated and 162 were downregulated in ETD-treated group. The Sketch heat maps of cluster analysis of difference comparison groups were shown in Fig. 2a. Detailed heat map was shown in supplementary Fig. 1.

\section{Bioinformatics analysis}

Two hundred sixty-one upregulated proteins in the vehicle treated model group were analyzed using GO enrichment analysis. Screening the three categories of GO entries with the number of corresponding proteins 





Table 1 Components and total synovitis score

\begin{tabular}{llll}
\hline & Con & Vehicle-CIA & ETD-CIA \\
\hline Inflammatory cell infiltration & $0.6 \pm 0.5$ & $3.4 \pm 0.7^{\# \#}$ & $1.4 \pm 0.5^{* *}$ \\
synovial cells proliferation & $0.2 \pm 0.5$ & $3.3 \pm 0.9^{\# \#}$ & $1.2 \pm 0.4^{* *}$ \\
neovascularization & $0.1 \pm 0.4$ & $2.1 \pm 0.8^{\# \#}$ & $0.9 \pm 0.6^{* *}$ \\
fibrous tissue proliferation & $0.6 \pm 0.5$ & $3.7 \pm 0.5^{\# \#}$ & $1.4 \pm 0.5^{* *}$ \\
Total synovitis score & $1.6 \pm 0.9$ & $12.6 \pm 2.3^{\# \#}$ & $3.6 \pm 1.0^{* *}$
\end{tabular}

Data are expressed as mean $\pm \mathrm{SD}(n=5), \# \#<0.01$ compared with control group; ${ }^{* *} p<0.01$ compared with Vehicle-CIA group

greater than 1 . According to the $-\log 10 p$ value of each entry, 10 entries are sorted from large to small. GO enrichment analysis of the top10 bar chart showed as Fig. 2. Among the upregulated proteins in vehicle treatment group, the top 10 terms of upregulated proteins in biological process category were "complement activation, classical pathway", "translation", "complement activation, alternative pathway", "fibrinolysis", "Arp2/3 complexmediated actin nucleation", "establishment of protein localization to chromatin", "AMP salvage", "blood coagulation", "fibrin clot formation" "nucleosome assembly" (Fig. 2b). Most of these terms were downregulated in ETD treatment group (Fig. 2c).

Protein-protein interaction enrichment analysis was analyzed by Metascape online analysis (https:// metascape.org/) and STRING online analysis (https:// string-db.org/). Functional links between proteins can usually be inferred from the genomic correlation between the genes that encode them. Two hundred sixtyone upregulated proteins in vehicle-treated model group were input, 245 of which were used for analysis. The interaction network diagram and cluster analysis are shown in Fig. 2d. According to the classification of Metascape, the differential proteins clustered in the following categories were highlighted: "Ribosome". "Posttranslational modification", "RNA splicing", "Actin nucleation", "Platelet degranulation", "Complement activation" and "Cellular response to stress". STRING online analysis results highlighted the cluster of complement and coagulation cascades, and platelet degranulation, and the proteins in this cluster was classified and marked with different color according to their properties (Fig. 2e).

KEGG (http://www.genome.jp/kegg/) is a database of biological systems that integrates genomic, chemical and systemic functional information. KEGG provides a reference knowledge base for linking genomes to life through the process of PATHWAY mapping, it is also the main public database for systematic analysis of protein metabolic pathways in cells. KEGG pathway classification results were shown in Fig. 3a. In KEGG Classification level1, the Genetic Information Processing pathway attracted our attention, a total of 111 upregulated proteins were enriched in this pathway, and 71 ones were downregulated in ETD treatment group. This pathway included several level2 pathway, such as "Transcription", "Translation", "Replication and repair" and "Folding, sorting and degradation".

KOG is clusters of orthologous groups for eukaryotic complete genomes, KOG analysis deeply studies the function and evolution pattern of eukaryotes. Two hundred sixty-one upregulated proteins in vehicle-treated model group were input, 210 of which were used for KOG function class analysis, and so that of 132 of 161 upregulated proteins in the ETD treatment group. As shown in Fig. 3b, according to the analysis results, a total of 24 items were listed, including "Posttranslational modification, protein turnover, chaperones", "Translation, ribosomal structure and biogenesis", "Signal transduction mechanisms", "General function prediction only", "Cytoskeleton", "RNA processing and modification", "Intracellular trafficking, secretion, and vesicular transport", "Lipid transport and metabolism", "Chromatin structure and dynamics", "Amino acid transport and metabolism", "Cell cycle control, cell division, chromosome partitioning", "Defense mechanisms", "Function unknown", "Carbohydrate transport and metabolism", "Nucleotide transport and metabolism", "Extracellular structures", "Inorganic ion transport and metabolism", "Transcription", "Cell wall/membrane/envelope biogenesis", "Energy production and conversion", "Replication, recombination and repair", "Secondary metabolites biosynthesis, transport and catabolism", "Cell motility" and "Nuclear structure Intracellular trafficking, secretion".

\section{ETD inhibited synovial inflammation, fibrosis and hyperplasia by downregulating complement and} coagulation cascades, and platelet degranulation cluster Combined with the results of Go and Metascape analysis, the complement and coagulation cascades, and platelet degranulation cluster obtained from STRING analysis attracted our attention, with a total of 21 proteins, including $\mathrm{Cfb}$, Serpinc1, Serping1, C8b, Apcs, LOC299282, Cpb2, C1s, C1qb, C9, Klkb1, Nhlrc2, Itih3, C5, Fgg, Hp, Fgb, C7, C8g, Tmsb4x, C3. Except C7, the rest of 20 proteins were down-regulated in ETD treatment group, they were showed in Fig. 4a.

The classical complement pathway activation index reflects the disease activity of rheumatoid arthritis [14]. In addition to complement members, several factors involved complement cascades were found upregulated in present study. A large number of immunoglobulins were upregulated in synovium tissue in CIA rats (Fig. 4a), which represented antibody mediated the classical pathway was activated in present study [15]. As reported, Collectin-12 (Colecl2), Apolipoprotein E (ApoE) and Plasma kallikrein (Klkb1) could trigger complement 


\section{a}

\section{KEGG Pathway Classification(V-vs-Cup)}

Brite Hierarchies--Protein families: signaling and cellular processes Brite Hierarchies--Protein families: metabolism.

Brite Hierarchies--Protein families: genetic information processing Cellular Processes--Transport and catabolism. Cellular Processes--Cellular community - eukaryotes. Cellular Processes--Cell motility .

Cellular Processes--Cell growth and death . Environmental Information Processing--Signaling molecules and interaction -

Environmental Information Processing--Signal transduction.

Genetic Information Processing--Translation .

Genetic Information Processing--Transcription . Genetic Information Processing--Replication and repair Genetic Information Processing--Folding, sorting and degradation .

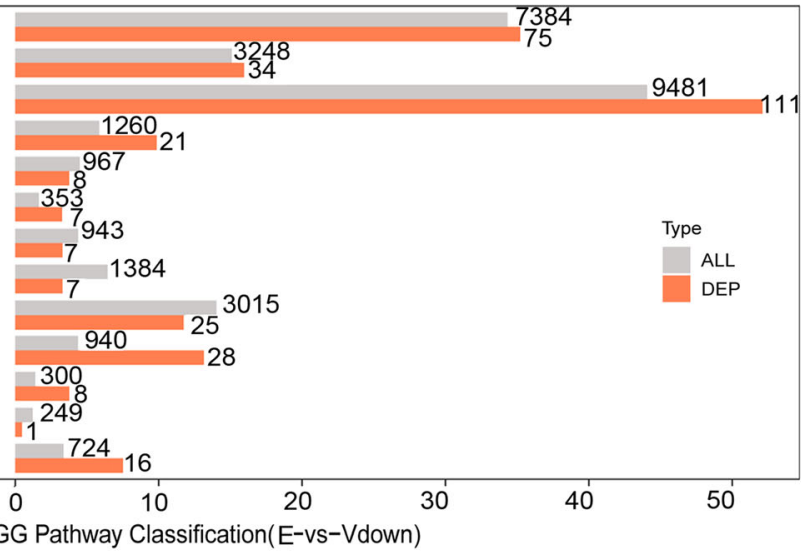

KEGG Pathway Classification(E-vs-Vdown)

Brite Hierarchies--Protein families: signaling and cellular processes . Brite Hierarchies--Protein families: metabolism

Brite Hierarchies--Protein families: genetic information processing Cellular Processes--Transport and catabolism. Cellular Processes--Cellular community - eukaryotes Cellular Processes--Cell motility .

Cellular Processes--Cell growth and death Environmental Information Processing--Signaling molecules and interaction

Environmental Information Processing--Signal transduction

Genetic Information Processing--Translation .

Genetic Information Processing--Transcription .

Genetic Information Processing--Replication and repair .

Genetic Information Processing--Folding, sorting and degradation.

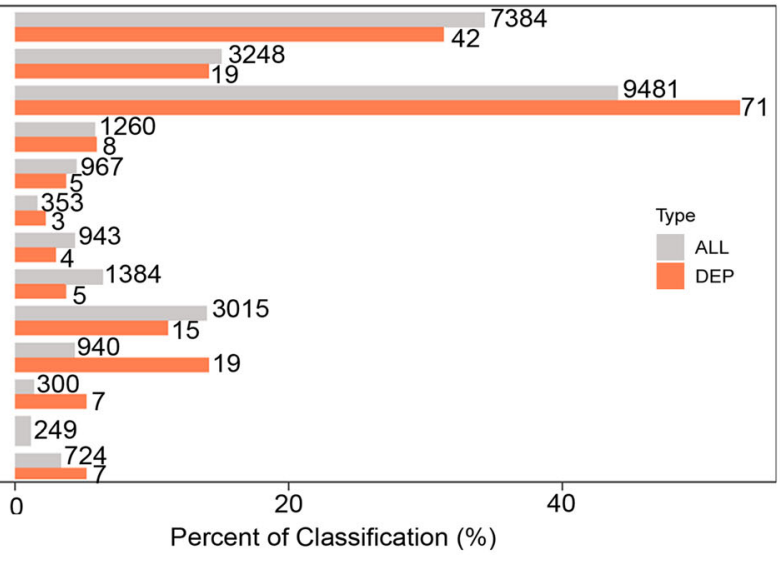

b

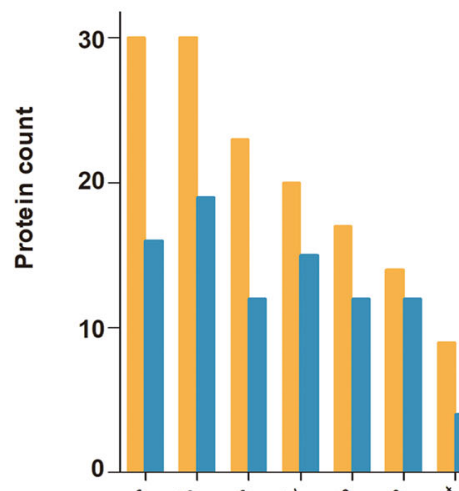

KOG function class

$V-v s-C$ up

E-vs-V down

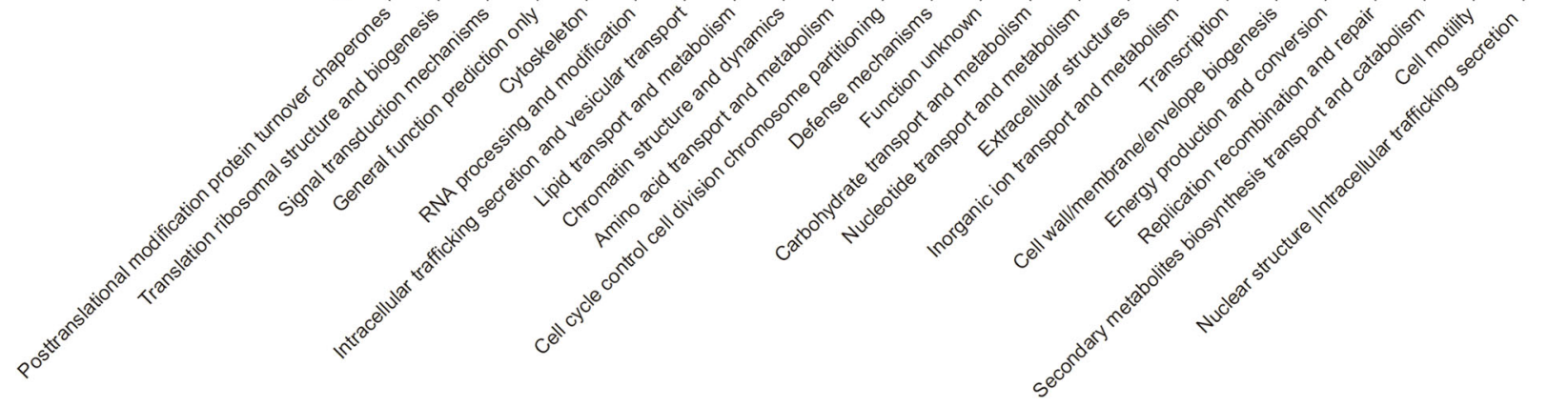

Fig. 3 (See legend on next page.) 
(See figure on previous page.)

Fig. 3 Visualizations of KEGG pathway classification and KOG function class analysis results. a Comparison of the distribution of differentially expressed proteins (DEP) and all proteins (ALL) at KEGG Level 2. The pathway to enrich the largest number of proteins is genetic information processing pathway. There are 111 proteins in upregulated proteins in Vehicle-CIA rats compared with control group, and 71 ones in downregulated proteins in ETD-CIA rats compared with Vehicle-CIA rats. b KOG function class analysis of upregulated proteins in Vehicle-CIA rats and downregulated proteins in ETD-CIA rats. C: Control group; V: Vehicle-CIA rats; E: ETD-CIA rats

pathway [16-19], they might also participate complement system activation in present study.

What is noteworthy is that the kallikrein-kinin system might also be involve in RA and may be the target of ETD. As reported, beside the control of prostaglandins by inhibiting COX2, ETD might also inhibit active oxygen generation and bradykinins formation [20]. FXIIa activates plasma prokallikrein (PK) to plasma kallikrein (PKA, Klkb1), which in turn activates FXII and the kallikrein kinin pathway, leading to local increase of bradykinin and further pro-inflammatory effects, such as vascular permeability, vasodilation and immune cell activation [21]. Here, the expression of Klkb1 was significantly upregulated in the synovium tissue of CIA rats, and ETD treatment significantly downregulated it, which indicated that ETD alleviated CIA might also by inhibiting kallikrein-kinin system.

In summary, the upregulation of immunoglobulins, Colecl2, Apoe, Klkb1 as well as a series of complement compartments C1s, C1qb, C3, C5, C7, C8g, C9 represented the activation of complement cascade in present study. As shown in Fig. 4b, a schematic diagram of upregulated proteins involved in complement system was constructed, in which those down-regulated proteins in ETD treatment group were marked with green borders.

Several components of the coagulation system were also upregulated, such as Fgb, Fgg and Thymosin $\beta 4$ $(\mathrm{Tmsb} 4 \mathrm{x})$. Fibrinogen $(\mathrm{Fg})$, as a downstream target of thrombin, is one of the most effective contributors of all coagulation proteins to inflammatory response. In addition to strong destructive tissue inflammation, fibrinogen seems to be the driving factor of chronic lowgrade inflammation. Fibrinogen- $\beta$ (Fgb) polymerizes with fibrinogen - $\alpha$ (Fga) and fibrinogen $-\gamma$ (Fgg) to form insoluble fibrin matrix and fibrin polymerization may be the key mechanism determining the pro-inflammatory ability of Fg. Fibrin is common in inflammatory foci, and extravascular fibrin deposition aggravates inflammation in a series of disease models [22].

Chronic inflammation leads to neovascularization and fibrous tissue proliferation, which all cause irregular synovial thickening. Fibrosis is a sign of excessive accumulation of collagen, which ultimately leads to organ failure [23]. In arthritis, synovial fibrosis might be the main reason of joint stiffness [24]. Here, hyperplastic synovium showed positive staining of Masson (Fig. 1e). The results of omics also showed that Collagen type IV alpha 1 chain (Col4a1), Collagen alpha-1(XI) chain (Col11a1) were upregulated in synovium tissue in CIA rats. Besides, another collagen related protein Lumincan (Lum) was also found to be upregulated. It was also found that one fibrosis factor Transforming growth factor- $\beta$ inducible protein (Tgfbi) was upregulated in the synovial tissue of CIA rats. In summary, members of the coagulation system (such as Fgg, Fgb) are related mediators of inflammatory processes, the upregulation of Fgb, Fgg, Tgfbi, Lum, CIV, CXI contributed to the synovial fibrosis, and ETD reduced synovial fibrosis by downregulating the expressions of Fgb, Fgg, Tgfbi, Lum and CXI (Fig. 4d).

It is noteworthy that Tmsb4 $\mathrm{x}$ has not been reported in RA so far, it might be a new target for RA treatment. Tmsb4x is a natural peptide with low molecular weight, which plays an important role in the repair and regeneration of damaged cells and tissues. After injury, platelets, macrophages and other cells release Tmsb4x to protect cells and tissues from further damage, reduce apoptosis, inflammation and microbial growth. But Tmsb4x also can bind to actin to promote cell migration, including mobilization, migration and differentiation of stem/progenitor cells, forming new blood vessels and regenerating tissues [25]. It could promote tumor proliferation and migration [26]. We suspected that it could also promote RA development and metastasis in the chronic stage of RA. ETD alleviated the syndrome of CIA rats might also through downregulating the expression of Tmsb4x.

\section{ETD inhibited synovial hyperplasia by downregulating proliferation related proteins}

Systems biology and its related bioinformatics are driving our understanding of central dogma and cell biology [27]. The central dogma refers to the transmission of genetic information from DNA to RNA, and then from RNA to protein, it includes several events, such as transcription, RNA editing, splicing, translation, posttranslational modification and DNA replication. The central dogma occurs in the reproductive process or cell proliferation, so the overexpression of central dogma related proteins might represent the prosperous proliferative activity. As shown in Fig. 3, compared to KEGG pathway classification, KOG functional analysis revealed the central dogma events more comprehensively. It showed that the 89 of 261 upregulated proteins were distributed in 


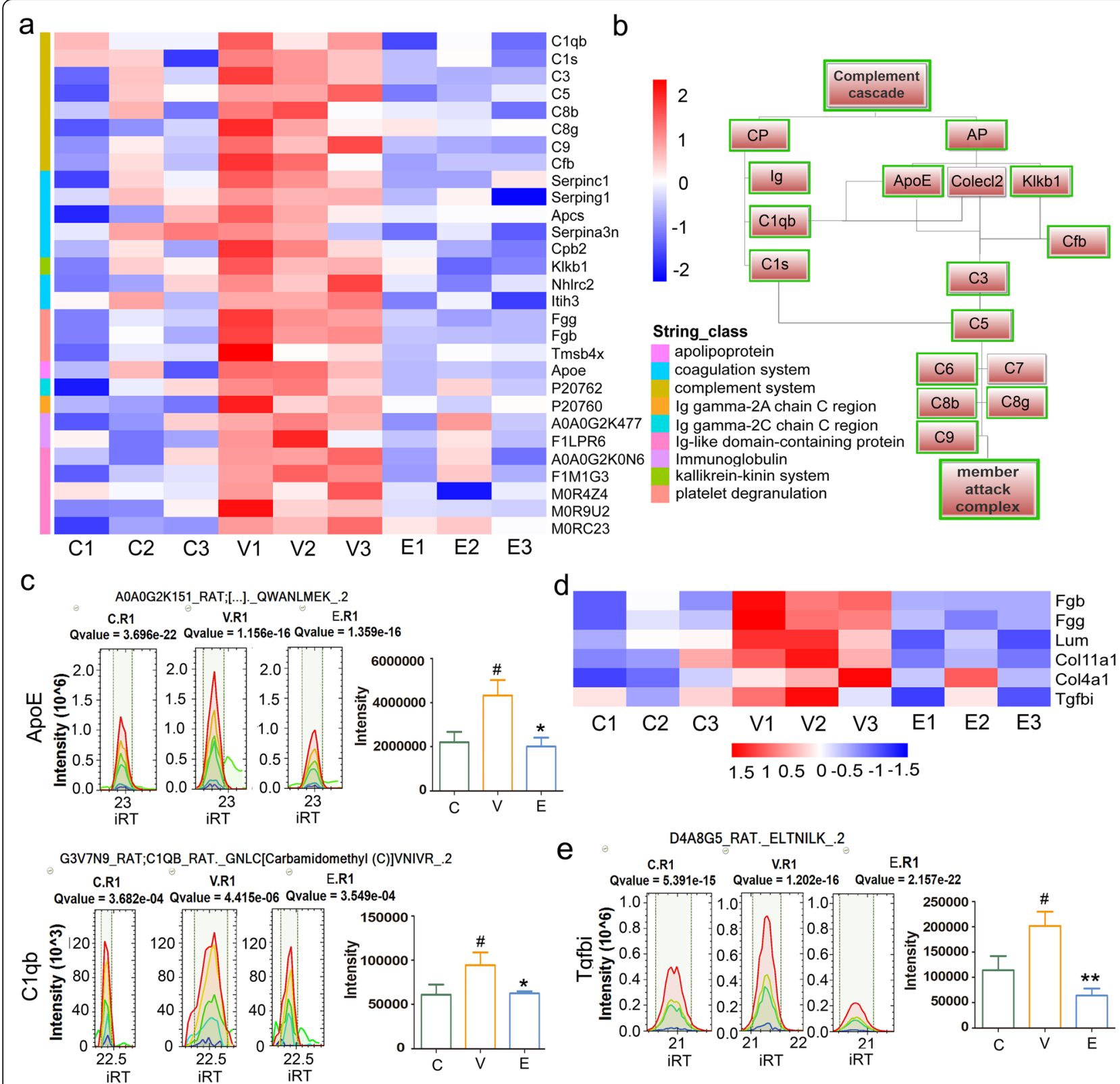

Fig. 4 The proteins enriched in cluster of complement and coagulation cascades, and platelet degranulation and their role in RA. a The heatmap of the proteins enriched in cluster of complement and coagulation cascades, and platelet degranulation as well as immunoglobulins. b Schematic diagram of up-regulated proteins involved in complement system. The upregulation of immunoglobulins, Colecl2, Apoe, Klkb1 as well as a series of complement compartments C1s, C1qb, C3, C5, C7, C8g, C9 represented the activation of complement cascade in present study, and those down-regulated proteins in ETD treatment group were marked with green borders. c The expression of ApoE and C1q were verified by PRM, the results were consistent with that of DIA. $\mathbf{d}$ The upregulation of Fgb, Fgg, Tgfbi, Lum, CIV, CXI contributed to the synovial fibrosis, and ETD reduced synovial fibrosis by downregulating the expressions of Fgb, Fgg, Tgfbi, Lum and CXI. e The expression of Tgfbi were verified by PRM, the result was consistent with that of DIA. \# $p<0.05$ compared with Control rats; ${ }^{*} p<0.05,{ }^{* *} p<0.01$ compared with Vehicle-CIA rats. C: Control group; V: Vehicle-CIA rats; E: ETD-CIA rats

all events of genetic central dogma and its extension, including "Transcription", "Chromatin structure and dynamics", "Replication, recombination and repair", "Cell cycle control, cell division, chromosome partitioning", "RNA processing and modification", "Translation, ribosomal structure and biogenesis", "Posttranslational modification, protein turnover, chaperones". They are shown in Fig. 5a and listed by their gene names. These proteins reflected the active proliferation of synovial tissue, for many of them were found involved in the abnormal proliferation of cancer cells. We carried a literature research on them, and their RA or cancer related 




Fig. 5 The differential proteins enriched in central dogma. a The heap map of 89 upregulated proteins in synovial tissue of vehicle-CIA rats compared with normal control rats. They were classified in the following events according to the KOG function analysis: "Cell cycle control, cell division, chromosome partitioning", "Chromatin structure and dynamics", "Transcription", "Replication, recombination and repair", "RNA processing and modification", "Translation, ribosomal structure and biogenesis", "Posttranslational modification, protein turnover, chaperones". b 59 downregulated ones in ETD-CIA rats compared with that in vehicle- treated rats. $\mathbf{c}$ The represent proteins in each event of central dogma was selected and verified by PRM method, including Pura, Nfic, Srrm2, Carhsp1 and Top2a, and the results were well consistent with that of DIA, which confirmed the accuracy of DIA proteomics results. \# $p<0.05$ compared with Control rats; ${ }^{*} p<0.05,{ }^{* *} p<0.01$ compared with Vehicle-CIA rats. C: Control group; V: Vehicle-CIA rats; E: ETD-CIA rats 
Table 2 Upregulated proliferation related proteins in RA related observations and cancer-related observations in synovial tissue of CIA rats

\begin{tabular}{|c|c|}
\hline Protein description & RA related observations \\
\hline \multicolumn{2}{|c|}{ DNA processing and modification } \\
\hline $\begin{array}{l}\text { Cyclic AMP-responsive } \\
\text { element-binding protein } \\
1 \text { (Creb1) }\end{array}$ & $\begin{array}{l}\text { Participated in in the movement, survival, proliferation and } \\
\text { chemokine/ cytokine synthesis of fibroblast like } \\
\text { synoviocytes [28] }\end{array}$ \\
\hline $\begin{array}{l}\text { Transcriptional activator } \\
\text { protein Pur-a (Pura) }^{a}\end{array}$ & - \\
\hline Nuclear factor I-C (Nfic) $)^{a}$ & - \\
\hline $\begin{array}{l}\text { RuvB-like 1(Ruvbl1, } \\
\text { Tip49) }^{\text {a }}\end{array}$ & - \\
\hline Histones & $\begin{array}{l}\text { Participated in a variety of autoimmune diseases including } \\
\text { arthritis [33] }\end{array}$ \\
\hline $\begin{array}{l}\text { Topoisomerase lla } \\
\text { (Top2a) }^{\text {a }}\end{array}$ & $\begin{array}{l}\text { Anti-Topo II a antibodies were detected in rheumatoid } \\
\text { arthritis [35] }\end{array}$ \\
\hline
\end{tabular}

\section{RNA processing and modification}

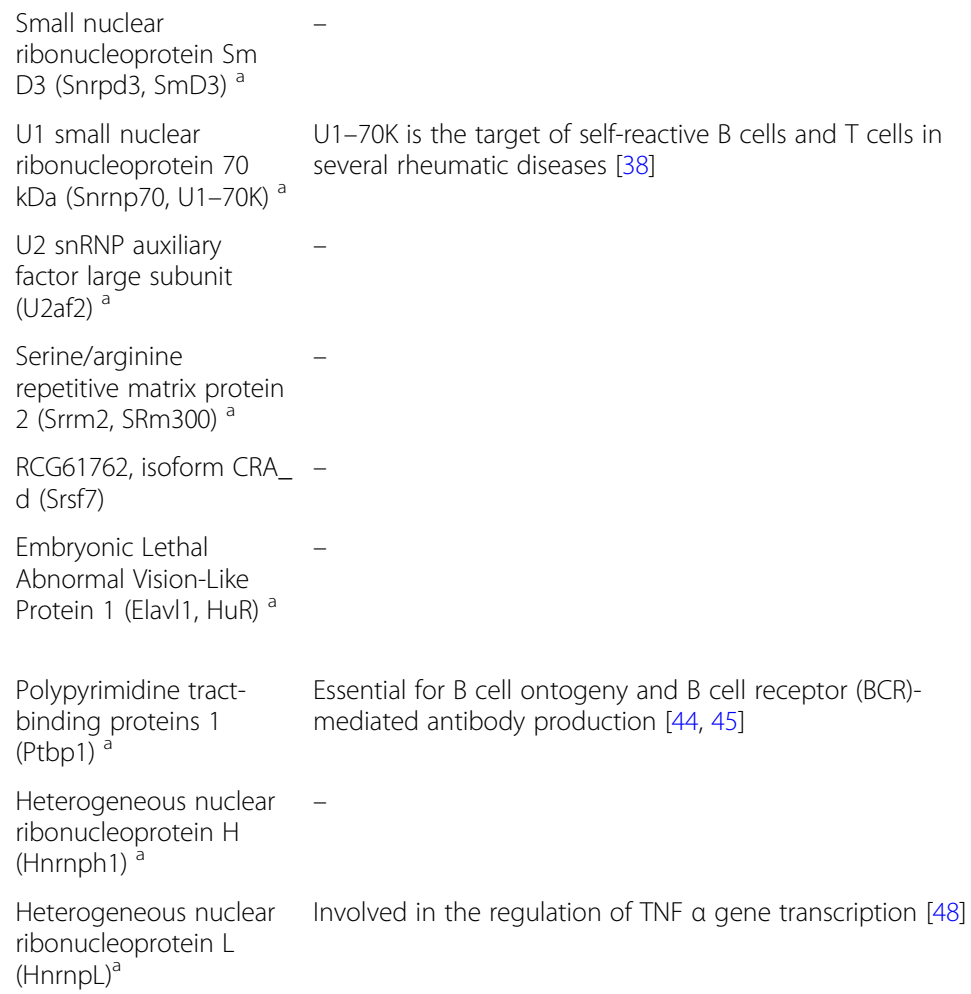

\section{Translation, ribosomal structure and biogenesis}

Tryptophanyl-tRNA

synthetase (Wars, TRPRS)

Tyrosyl- tRNA synthetase -

(Yars)

Basic leucine zipper and -

W2 domains 2 (Bzw2)

Multiple copies in T-cell -

malignancy $1(\text { Mcts } 1)^{a}$

Eukaryotic initiation factor 4B (Eif4b)

\section{Cancer related observations}

Involve in the migration and proliferation of cancer cells [29]

Assist DNA repair after DNA damage [30]

Promote the transcription of IncRNA LBX2-AS1 in gastric cancer (GC) cells [31]

Modulated wnt/ $\beta$-catenin activation [32]

Histone modifications are the targets of epigenetic drugs against cancer [34]

The abnormal changes of Top2a and its interacting proteins and their modifications may play a key role in human cancer [36]

As inhibitor of p53 activity in multiple NSCLC cell lines [37]

With robust and consistent expression levels across breast cancers [39]

Promote the Warburg effect and tumorigenesis [40]

Silence Srrm2-AS can block cell cycle and promote cell apoptosis [41]

SRSF7 knockdown promotes apoptosis of colon and lung cancer cells [42]

Enhance cell proliferation and survival, promote angiogenesis, evading the recognition of the immune system, and increase the invasion and metastasis potential of cancer cells [43]

Promote the proliferation and metastasis of cancer [46]

Increased expression of hnrnph1 is associated with poor prognosis of Mantle cell lymphoma [47]

As a prostate cancer dependency regulating RNA splicing [49]

Possible contributor during uveal melanoma progression [50]

Promotes gastric cancer progression through activating PI3K-Akt signaling [51]

Promote the malignant progression of CRC (Colorectal cancer) via activating ERK/MAPK signaling [52]

Promoting cell proliferation, inhibiting apoptosis and promoting angiogenesis [53]

The depletion of elF4B in cancer cells can reduce cell proliferation [54] 
Table 2 Upregulated proliferation related proteins in RA related observations and cancer-related observations in synovial tissue of $\mathrm{ClA}$ rats (Continued)

\begin{tabular}{|c|c|c|}
\hline Protein description & RA related observations & Cancer related observations \\
\hline Ribosomal proteins ${ }^{a}$ & $\begin{array}{l}\text { Ribosomal proteins were upregulated in the synovial } \\
\text { membranes of female patients with Osteoarthritis [55] }\end{array}$ & $\begin{array}{l}\text { Cope with increased protein synthesis and maintain } \\
\text { unrestricted growth of cancer cell [56] }\end{array}$ \\
\hline $\begin{array}{l}\text { Calcium-regulated heat } \\
\text { stable protein } 1 \\
\text { (Carhsp1) }\end{array}$ & Regulates the stability of TNF -a mRNA [57] & $\begin{array}{l}\text { Phosphorylation of CARHSP1 } 1^{\mathrm{S} 30, \mathrm{S32}} \text { could be potential } \\
\text { candidates associated with } \mathrm{K} 8 \text { phosphorylation mediated } \\
\text { tumorigenicity [58] }\end{array}$ \\
\hline
\end{tabular}

Posttranslational modification, protein turnover, chaperones

\begin{tabular}{|c|c|}
\hline cathepsin D (Ctsd) & Contributed to the pathogenesis of RA [59] \\
\hline cathepsin $\mathrm{H}(\mathrm{Ctsh})^{a}$ & - \\
\hline $\begin{array}{l}\text { Lysosome-associated } \\
\text { membrane glycoprotein } \\
1 \text { (Lamp1) }\end{array}$ & - \\
\hline $\begin{array}{l}\text { Dipeptidyl peptidase } 2 \\
\text { (Dpp7) }^{\text {a }}\end{array}$ & - \\
\hline Proteasome $^{a}$ & $\begin{array}{l}\text { Proteasome inhibitors (PIs) can inhibit the activation of } \\
\text { nuclear factor (NF)-kB and transcriptional regulation of pro- } \\
\text { inflammatory cytokine release, and/or (b) induce apoptosis } \\
\text { of activated immune cells [64] }\end{array}$ \\
\hline $\begin{array}{l}\text { Glutathione peroxidase } \\
(\text { Gpx } 1)\end{array}$ & $\begin{array}{l}\text { Aberrant expression of Gpx contributed to the etiology of } \\
\text { rheumatoid arthritis (RA) [66] }\end{array}$ \\
\hline $\mathrm{HSP} \mathrm{O}^{\mathrm{a}}$ & $\begin{array}{l}\text { Induce Th17 frequency and Th17 /Treg ratio in vivo, } \\
\text { reflecting its pro-inflammatory activity [68] }\end{array}$ \\
\hline $\begin{array}{l}\text { Heat shock protein } 27 \\
\left(\mathrm{Hspb7}^{\mathrm{a}}\right.\end{array}$ & Significantly increased in RA synovial tissues [70] \\
\hline $\begin{array}{l}\text { Prostaglandin E synthase } \\
3{\text { (Ptges } 3, \mathrm{p} 23)^{\text {a }}}^{\text {Pat }}\end{array}$ & - \\
\hline
\end{tabular}

Cell cycle control, cell division, chromosome partitioning

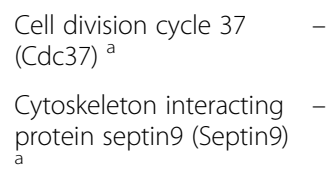

Ctsd is a tumor-specific extracellular target in TNBC Triplenegative breast cancer (TNBC) [60]

Promoted cancer cell progression by affecting integrin activation and adhesion strength [61]

Supported tumor growth and metastasis [62]

Highly expressed in Multiple myeloma [63]

A multifaceted target for anti-cancer therapies [65]

Gpx 1 is commonly overexpressed in most human cancers and can be used as a prognostic biomarker for identifying cancer types [67]

Promoted tumorigenesis in many kinds of cancer [69]

Protected cancer cells from apoptosis inducing agents [71]

Beneficial to the abnormal proliferation of cancer cells [72]

Promoting cancer cell proliferation, G1-S conversion and inhibiting apoptosis [73]

Has a carcinogenic effect and is an oncogene [74]

Promotion of migration and invasion of cancer cells [75] a: Downregulated in ETD-CIA group; -: No retrieved

observations were summarized in Table 2. Among these 89 up-regulated proteins, 59 were downregulated in ETD treatment group (Fig. 5b), which indicated the inhibition of the active proliferation of synovial tissue.

\section{PRM proteomics validation}

We selected some represent proteins in the terms of complement activation and fibrosis and verified them by PRM method. The expression of ApoE, C1q and Tgfbi were consistent with that of DIA (Fig. $4 \mathrm{c}$ and e). According to KOG function class analysis, the represent proteins in each event of central dogma were verified by PRM method, including Pura, Nfic, Srrm2, Carhsp1 and Top2a, the results were well consistent with that of DIA (Fig. 5c), which the confirmed the accuracy of DIA proteomics results.

\section{The safety of ETD treatment}

The safety of 7 months treatment of ETD was investigated by blood biochemistry analysis. Compared with normal control rats and vehicle-CIA rats, no obvious abnormality was found in biochemical tests in ETD-CIA rats. It indicated that long term administration of ETD for 7 months had no toxic reactions such as liver and kidney, which further proved the safety of ETD. The results were listed in Table 3.

\section{Discussion}

It is well known that ETD can reduce pain and inflammation by inhibiting COX2, and it has pain and joint swelling relief effects on RA [8, 76, 77]. But it is generally believed that NSAIDs do not have the same therapeutic effect as DMARDs. Our study provided evidence 
Table 3 The results of blood biochemical tests

\begin{tabular}{lllll}
\hline & & Con & Vehicle-CIA & ETD-CIA \\
\hline Glu-G & $\mathrm{mmol} / \mathrm{L}$ & $10.72 \pm 1.25$ & $11.74 \pm 6.43$ & $9.43 \pm 4.23$ \\
T-Bil-V & $\mu \mathrm{mol} / \mathrm{L}$ & $0.58 \pm 0.21$ & $0.19 \pm 0.40$ & $0.21 \pm 0.27$ \\
ALT & $\mathrm{U} / \mathrm{L}$ & $49.25 \pm 3.90$ & $57.90 \pm 18.78$ & $63.56 \pm 36.47$ \\
AST & $\mathrm{U} / \mathrm{L}$ & $133.68 \pm 22.53$ & $128.67 \pm 26.01$ & $120.19 \pm 56.61$ \\
ALP & $\mathrm{U} / \mathrm{L}$ & $97.75 \pm 26.75$ & $153.28 \pm 93.99$ & $97.90 \pm 41.24$ \\
Y-GT & $\mathrm{U} / \mathrm{L}$ & $0.90 \pm 0.36$ & $1.24 \pm 0.88$ & $1.00 \pm 0.44$ \\
TP & $\mathrm{g} / \mathrm{L}$ & $53.43 \pm 2.11$ & $56.94 \pm 3.44$ & $55.43 \pm 19.68$ \\
TG & $\mathrm{mmol} / \mathrm{L}$ & $0.75 \pm 0.19$ & $0.63 \pm 0.16$ & $0.72 \pm 0.28$ \\
LDL-C & $\mathrm{mmol} / \mathrm{L}$ & $0.67 \pm 0.04$ & $0.59 \pm 0.11$ & $0.68 \pm 0.24$ \\
HDL-C & $\mathrm{mmol} / \mathrm{L}$ & $1.85 \pm 0.13$ & $1.75 \pm 0.26$ & $1.78 \pm 0.59$ \\
TC & $\mathrm{mmol} / \mathrm{L}$ & $2.39 \pm 0.17$ & $2.28 \pm 0.28$ & $2.42 \pm 0.83$ \\
UREA & $\mathrm{mmol} / \mathrm{L}$ & $5.97 \pm 0.35$ & $6.36 \pm 1.07$ & $6.69 \pm 2.31$ \\
CK-MB & $\mathrm{U} / \mathrm{L}$ & $860.65 \pm 406.12$ & $711.17 \pm 457.68$ & $738.49 \pm 401.94$ \\
CK & $\mathrm{U} / \mathrm{L}$ & $430.93 \pm 175.77$ & $457.21 \pm 215.77$ & $382.81 \pm 202.93$ \\
LDH & $\mathrm{U} / \mathrm{L}$ & $1099.15 \pm 481.02$ & $877.71 \pm 532.64$ & $909.40 \pm 459.93$ \\
ALB II & $\mathrm{g} / \mathrm{L}$ & $29.10 \pm 1.38$ & $29.40 \pm 0.80$ & $29.43 \pm 10.38$ \\
CREA-S & $\mu \mathrm{mmol} / \mathrm{L}$ & $35.98 \pm 2.65$ & $34.76 \pm 5.29$ & $34.07 \pm 13.54$ \\
\hline Data & &
\end{tabular}

Data are expressed as mean \pm SD $(n=8)$; there were no significant differences among three groups

that ETD also has disease modifying anti rheumatic effect. It could reduce primary and secondary lesions of CIA rats by inhibiting synovial inflammation, fibrosis and hyperplasia. Moreover, with the help of proteomic technology and bioinformatics analysis, the molecular mechanisms of synovial hyperplasia in RA were explored, and the mechanisms hidden behind the protective effects of ETD on RA were further investigated.

Our study for the first time to identified so many differential proteins in the synovium of CIA rats, we focused on the upregulated proteins and tried to clarify their roles in RA. STRING analysis highlighted the cluster of complement and coagulation cascades, and platelet degranulation, it involved in synovial inflammation and fibrosis. ETD treatment downregulated most of proteins in this cluster. Our study was consistent with previous research views that the activation of classical pathway $(\mathrm{CP})$ and alternative pathway (AP) are involved in RA [78]. As reported, the main component of Rheumatoid factor (RF) is IgM, including IgG, IgA and IgE. RF-IgG complex in synovium can fix and activate complement, produce $\mathrm{C} 3 \mathrm{a}$ and $\mathrm{C} 5 \mathrm{a}$, and attract neutrophils and monocytes to secrete. Neutrophils, monocytes and synoviocytes (type A cells) phagocytize immune complexes, then activate and release lysosomal enzymes, including neutral protease, collagenase and various mediators, such as prostaglandins, leukotrienes and IL-1, leading to the destruction of synovium and articular cartilage [79]. A large number of immunoglobins were found upregulated, they activated CP. Some other factors of complement activation were also found in present study, for example, the upregulations of AopE, Colecl2, Klkb1 also participated the activation of complement system in RA.

The activation of the immune system has been increasingly considered to be related to the procoagulant state of patients with RA. Many different components of the hemostatic system (such as thrombin, fibrinogen, coagulation factor XIII and fibrinolytic system factor) are related mediators of inflammatory process and inflammation control [80]. Furthermore, fibrinogen promoted renal fibrosis by inducing fibroblast proliferation and activating TGF- $\beta 1 /$ pSMAD2 signaling [81]. Here, the upregulations of components of the hemostatic system (Fgg, $\mathrm{Fgb}$ ) participated in inflammation, the upregulation of Fgb, Fgg, Tgfbi, Lum, CIV, CXI contributed to the synovial fibrosis.

As reported, the immune complement system was involved in mucinous cystic neoplasm $(\mathrm{MCN})$, combined with their performance in this study, it supported the inference that the similarity between cancer and RA [82]. Moreover, with the help of KOG analysis, this inference got more supported. According to KOG analysis, the active proliferation of synovial tissue was also reflected by the 89 upregulated proliferation related proteins, As shown in Fig. 5, these proteins were classified according to their roles in central dogma. Among these 89 upregulated proteins, 59 ones were downregulated in ETD treatment group, which indicated that ETD alleviated the symptom of RA by reducing these proliferation related proteins. We conducted a literature survey for them, and their RA and cancer related observations were listed in Table 2, and classified them by their role in the events of central dogma.

Transcription and replication are two important processes of cell survival and proliferation. Although these two processes are two different events, they are inextricably linked because they share certain proteins [83]. So, several proteins involved in "Transcription", "Chromatin structure and dynamics", and "Replication, recombination and repair" were integrated into DNA processes and modifications category in Table 2, including Creb1, Nfic, Pura, Ddb1, Ruvbl1, histones and Top2a. Their upregulations indicated that gene transcription and replication were significantly promoted in the synovium of CIA rats. They might be the main factors to ensure a high level of transcription and replication, and the way to break or inhibit them may be effective strategy for RA.

Transcription control is the first step in the formation of immune response. RNA editing, localization, stability and translation are other key steps in the final gene expression after transcription. The human genome consists 
of about 20,000 genes encoding proteins, but the transcriptome and proteome are much larger. This result is due to RNA splicing, which converts precocious mRNA into mature protein encoded mRNA by removing intron sequence. RNA splicing is a dynamic reaction catalyzed by a ribonucleoprotein complex called spliceosome, which involves the sequential recruitment and release of many different proteins in pre mRNA transcripts. RNA splicing also has regulatory mechanisms, and if something goes wrong, diseases like cancer or autoimmune diseases can occur [84]. The analysis based on KOG function class showed that 14 proteins upregulated proteins were involved in the category of RNA processing and modification. Among them, Snrpd3, Snrnp70, U2af2, Alyref, Srsf7 and Srrm2 are components of the RNA splicing machinery. RNA binding proteins (RBPs) play regulatory role in post transcriptional processes by acting on regulatory sequences, resulting in differences in mRNA abundance and protein expression of cytokines [85]. In present study, the upregulation of RBPs included HuR (Elavl1), Ptbp1. Among14 upregulated RNA related proteins in synovial tissue of CIA model, 12 of them were downregulated in ETD treatment group, they are Alyref, Ddx17, Elavl1, Hnrnph1, Hnrnpl, Ptbp1, Snrnp70, Snrpd3, Srrm2, U2af2, Ncl, and Pabpc1. Furthermore, as shown in Table 3, according to literature research, most of above RNA related proteins played roles in the occurrence and development of tumors. Some tumors are highly dependent on splicing function to maintain survival, which makes RNA related events become target for cancer treatment [86]. Among these proteins, U1-70K (Snrnp70) is the target of self-reactive $B$ cells and $T$ cells in several rheumatic diseases [38], Ptbp1 is essential for B cell ontogeny and B cell receptor (BCR)-mediated antibody production [44, 45], HnrnpL involved in the regulation of TNF $\alpha$ gene transcription [48]. Only these three proteins might be associated with RA, except this, there's nothing more evidences about these RNA processing and modification proteins are related with RA. We proposed the rest of RNA-related proteins might also be involved in the synovial hyperplasia, and they might be new targets of RA treatment.

According to the KOG function class analysis, 28 upregulated proteins were specifically related to translation, ribosomal structure and biogenesis, including Gars, Wars, Yars, Gar1, Bzw2, Carhsp1, Mct-1, Eif4b, 40S ribosomal protein (S3a, S4, S5, S7, S9, S17, S25, S26) and $60 \mathrm{~S}$ ribosomal proteins (L22, L23, L23a, L24, L27a, L35, L37a, L6). They also related with cancer progression, and their cancer relevant observations were showed in Table 2. They might not only cope with increased protein synthesis and maintain unrestricted growth of cancer, but also account for the increased protein synthesis in synovial proliferation in CIA rats. Among them,
Ribosomal proteins were upregulated in the synovial membranes of female patients with Osteoarthritis [55], Carhsp1 can regulate the stability of TNF $-\alpha$ mRNA [57], they might be associated with RA, no other proteins, such as Wars, Yars, Bzw2, Mcts1 and Eif4b were reported in RA. 17 of 28 up-regulated proteins were downregulated in ETD-CIA rats indicated protein synthesis was inhibited by ETD treatment.

After protein translation, further posttranslational modification, protein turnover and chaperones are needed to ensure protein function. According to the functional analysis of KOG, 30 related proteins are up regulated, including lysosomal enzymes Ctsd, Ctsh, Lamp1 and Dpp7, proteasome related proteins Psmb2, Psmb5, Psmc4, Psmd1 and Psmd3, protein turnover and chaperones Hspa12b, Hspa1a, Hspa2, Hsp27, Ptges3 and so on. Lysosomal cathepsins play an important role in the induction and diagnosis of RA. The levels of several tissue proteases (B, D, G, K, l and S) in serum and synovial fluid of patients are considered as the basis of RA diagnosis, regulating cathepsin activity can improve the pathological characteristics of some autoimmune and inflammatory diseases [87]. A study showed that the activities of cysteine protease Ctsb, Dpp1, aspartic protease Ctsd and two glycosidases are increased in all patients, which is related to the progress of RA [88]. Several cathepsin inhibitors (B, D, l, K and S) have been described and their activity in rheumatic autoimmune diseases has been evaluated [89]. In present study, 16 lysosomal enzymes (Ctsa, Ctsb, Ctsc, Ctsd, Ctsh, Ctss, Dpp3, Dpp4, Dpp7, Acp2, Scarb2, Lamp1, Lamp2, Lyz2, Tpp1, and Tpp2) were identified and quantified (Supplementary Table 2), among them, Ctsd, Ctsh, Lamp1 and Dpp7 were significantly upregulated in CIA rats, and Ctsh, Dpp7 were significantly downregulated by ETD treatment. Although many lysosomal enzymes were reported in RA, but Ctsh and Dpp7 were not yet found. As shown in Table 2, Ctsd and Ctsh was reported to promote cancer cell progression $[60,61]$.

The $26 \mathrm{~S}$ proteasome plays a key role in maintaining protein homeostasis by removing misfolded or damaged proteins that may impair cell function, and removing proteins that are no longer needed. This type of proteolysis is necessary in many ways, including spermatogenesis or generation of a subset of MHC class I-presented antigenic peptides. Many diseases, such as neurodegenerative diseases and cancer are closely related to the activity of proteasome, which makes it an important pharmacological target [65]. The 26S proteasome is composed of two $19 \mathrm{~S}$ regulatory particles and $20 \mathrm{~S}$ core proteasome complex. Within the $20 \mathrm{~S}$ core complex, Psmb5 displays a chymotrypsin-like activity. Bortezomib is the first-class proteasome specific drug approved by FDA, which can reversely inhibit chymotrypsin activity 
of $20 \mathrm{~S}$ proteasome [90]. The drug has been approved for the treatment of multiple myeloma, recurrent/refractory multiple myeloma and mantle cell lymphoma. In addition, it can also be used in autoimmune diseases such as RA. In present study, Psmb2, Psmb5, Psmc4, Psmd 1 and Psmd 3 were up-regulated in CIA rats, and Psmb5 and Psmc4 were significantly decreased in ETD treatment group, which indicated the activity of proteasome was decreased by ETD treatment.

Hsp70, Hsp40, Hsc70, Cdc37, p23 (Ptges3) and immunophilins are different cochaperones of Hsp90, which indispensable in achieving different functions and selectivity through direct protein-protein interactions with Hsp90 [91]. In present study, 13 PPIases were identified (Supplementary Table S2), among them Ppic, Fkbp7 and Fkbp9 were upregulated in CIA rats, all of them were significantly downregulated in ETD treatment group. It is well known that Hsp27, Hsp70, Hsp90 and other chaperone proteins are often enhanced in the process of tumorigenesis, and play an important role in many key steps of tumorigenesis and development [92, 93]. In present study, Heat shock protein family A (Hsp70) member 12B (Hspa12b), Heat shock $70 \mathrm{kDa}$ protein 1A (Hspa1a), Heat shock-related $70 \mathrm{kDa}$ protein 2 (Hspa2) were found upregulated, they belong to molecular chaperones Hsp70/ Hsc70, Hsp70 superfamily. Hsp27 (Hspb7) and the cochaperones of Hsp90 (Ptges3, PPIases) were also upregulated, Hspa2, Hspb7, Ppic and Ptges3 were downregulated in ETD treatment group. So far, there are some conflicting conclusions about the role of Hsp70 in RA. Some studies think that it has pro-inflammatory effect, and other think it has anti-inflammatory effect [68]. We think that the role of Hsp70 may be different from the stage of RA, but in the chronic stage, such as this study, the role of Hsp70 should be to promote proliferation, just like its role in tumor. The upregulations Hsp27, Hsp70, and Hsp90 cochaperones might all contribute to synovial hyperplasia, which was another evidence for synovial hyperplasia with tumor proliferation characteristics.

The above series of activities and associated proteins are preparing for cell proliferation. Following these events, cells will enter into the stage of division and proliferation. In present study, the final result of cell proliferation is synovial hyperplasia. There are also some cell division related proteins in the upregulated protein. Cdc37 (p50) and Septin9 are two belong to the catalog of cell cycle control, cell division, chromosome partitioning. They were both involved in tumorigenesis and invasion, and their cancer relevant observations were also listed in Table 2. It should be noted that Septin9 has a carcinogenic effect and is an oncogene an oncogene, which might be associated with RA related cancer. The expression of Cdc37 and Septin9 were both downregulated by ETD treatment.
As reported, the gradual formation of hypoxia and malnutrition microenvironment in RA joints are similar to that of solid tumors [94]. Some markers of RA associated with malignancies have been identified [95]. Here, we provided the evidence at the molecular level that the process of cell proliferation was also very similar between cancer and RA. In other words, tumor and arthritis might have common therapeutic targets, and proteasome inhibitor is a good example [90]. As shown in Table 3, the literature research showed that most of these proteins are related to the abnormal proliferation and metastasis of tumor cells, but few of them have been studied in RA, several proteins were firstly reported associated with RA, such as Pura, Nfic, Ruvbl1, Snrpd3, U2af2, Srrm2, Srsf7, Elavl1, Hnrnph1, Wars, Yars, Bzw2, Mcts1, Eif4b, Ctsh, Lamp1, Dpp7, Ptges3, Cdc37 and Septin9. They may be potential targets for RA, but still need a lot of validation work. The downregulation of these proteins was beneficial for the remission of RA, which was confirmed in ETD treatment group.

As a selective COX-2 inhibitor, more and more studies have shown that ETD has other functions besides analgesia and anti-inflammatory. The overexpression of COX2 was also involved in tumor regeneration, and ETD showed anti-proliferative effects by suppressing COX-2 in cancer $[9,10]$. Due to genetic susceptibility, persistence of long-term disease activity under persistent immune stimulation and anti-RA therapy, especially the use of DMARDs, the risk of certain cancers in RA patients would increase, such as lung cancer, lymphoma, non-melanoma skin cancer, and so on [96-98]. As shown in Table 2, so many upregulated proteins in CIA rats in present study were related with cancer, most of them can promote cancer cell proliferation and metastasis, some even have carcinogenic effects. This might be the mechanism of the occurrence of RA related cancer. And their downregulation in ETD treatment group also provided the clue that ETD might also have the potential of preventing tumor occurrence in patients with RA. We hypothesized that ETD alone or combined with DMAR Ds in the treatment of RA may reduce the risk of cancer, but evidence is still needed to confirm this.

We also investigated the safety of long-term administration ETD. Biochemical results showed that there was no abnormal biochemical index after taking ETD for 7 months, which further validated the safety of ETD.

In conclusion, using DIA and PRM proteomics to study the mechanism of synovial proliferation provided more clues for the anti-synovial hyperplasia therapy. ETD can not only relieve pain and inflammatory but also has disease modifying effect in CIA rats by inhibiting synovial inflammation, fibrosis and proliferation. Longterm use of ETD in RA patients has a good efficacy and safety, it still has important clinical value. 


\section{Materials and methods}

\section{Animals and reagents}

A total of 24 6-7-week-old healthy male Wistar rats (SPF grade) with body weights of $160-180 \mathrm{~g}$ was purchased from Vital River Laboratory Animal Co. Ltd. (Beijing, China). The rats were housed in a clean room at a temperature of $23 \pm 2{ }^{\circ} \mathrm{C}$ and a humidity of $50 \pm 5 \%$ with a $12 \mathrm{~h}$ alternating light and dark cycle. They were permitted free access to food and water. All animal experiments were performed according to the National Institutes of Health Guidelines for the Care and Use of Laboratory Animals and were approved by the Animal Care and Use Committee of Shandong Province, China. ETD was provided by national engineering and technology research center of chirality pharmaceutical, Lunan Pharmaceutical Group Co. Ltd.

\section{Animal treatment}

Collagen-induced arthritis (CIA) was induced in rats by intradermal injections in right hindfoot base with $100 \mu \mathrm{g}$ of bovine collagen type II (CII) $\left(2 \mathrm{mg} . \mathrm{mL}^{-1}\right.$ ) (Chondrex, Inc., USA) emulsified in complete Freund's adjuvant (CFA) (2 mg. $\mathrm{mL}^{-1}$; Chondrex, Inc., USA). Eight rats served as control group by intradermal injections in right hindfoot base with saline. Two weeks after immunization, 16 rats with the right heel width $>12 \mathrm{~mm}$ were selected as the successful model rats, they were randomly divided into two groups $(n=8)$. One group was considered as vehicle- CIA group (V) that received vehicle $(0.5 \%$ sodium carboxymethyl cellulose, $\mathrm{CMC}-\mathrm{Na})$ $(\mathrm{n}=8)$; another group was ETD-CIA group (E) received ETD $\left(5 \mathrm{mg} \cdot \mathrm{kg}^{-1}\right)$ daily as a single dose by gavage for 7 months $(n=8)$, ETD powder was added into $0.5 \%$ sodium carboxymethylcellulose and homogenized for administration. $0.5 \% \mathrm{CMC}-\mathrm{Na}$ was given to the control group $(C)(n=8)$. All outcome measurements were made by observers blinded to treatment group.

At the end of the experiments, the animals were killed after anesthetization with an intraperitoneal injection of sodium pentobarbital $\left(50 \mathrm{mg} \cdot \mathrm{kg}^{-1}\right)$. Blood was taken from abdominal main vein. The serum was separated from the blood samples by centrifugation at $1000 \mathrm{~g}$ for $10 \mathrm{~min}$ at $4{ }^{\circ} \mathrm{C}$ and taken for biochemical analysis. Synovium of right posterior knee joint in each group were taken after bloodletting, three synovium tissues stored at $-80{ }^{\circ} \mathrm{C}$ for further proteomics analysis, and five synovium tissues were fixed in $10 \%$ formalin for morphological analysis.

\section{Assessment of joint arthritis}

The articular index was calculated at $0,0.5,1,2,3,4,5$, 6 and 7 months after ETD treatment. The followed scoring system were used to assess the severity of arthritis: 0 , normal, no evidence of swelling; 1 , mild swelling of the toe joint; 2, moderate swelling extending from the toe joint to the ankle; 3 , severe swelling extending from the ankle to metatarsal joints; and 4, extreme swelling and deformation of toe joint, the ankle and metatarsal joints. The cumulative score for all four paws of each rat was used as arthritis score (maximum of 16 per rat) to represent overall disease severity and progression. The right heel width was measured with caliper to quantify the swelling in all groups.

\section{Pain behavior}

Punctate allodynia was measured by Ugo Basile Dynamic Plantar Aesthesiometer 37,450. At each paw withdrawal, the Dynamic Plantar Aesthesiometer automatically detects and records actual force at the time of paw withdrawal reflex, and the force is used to indicate the pain threshold.

\section{$\mathrm{HE}$ and Masson staining}

Fixed synovium tissue were processed and embedded in tissue embedding rings (Tissue-Tek ${ }^{\odot}$ TEC $^{\mathrm{m}}$ 5.Sakura Fine tek Japan CO., Ltd). Sections of $4 \mu \mathrm{m}$ in thickness were subjected to haematoxylin and eosin (HE) staining by automatic staining machine (Leica ST5020). Paraffin sections $(4 \mu \mathrm{m})$ were carried out for Masson staining. Masson staining kit (Nanjing Jiancheng Bioengineering Institute, Nanjing, Jiangsu, China) was used for Masson staining according to the manufacturers' instructions. Under the light microscope, the nuclei were dark blue, collagen fibers, cartilage and mucus were blue, muscle fibers, cellulose and red blood cells were red. Slides were viewed and captured by PannoramicViewer1.15.4 (3DHISTECH. Ltd). Synovitis evaluation was refined according to the Krenn's score [99]. Assessing in four components: inflammatory cell infiltration, synovial cells proliferation, neovascularization and fibrous tissue proliferation. Each item was evaluated by two blind observers using a subscale of $0-4$ points, where 0 indicated absence, 1 mild, 2 moderate, 3 severe and 4 extreme. The total synovitis score (TSS) was calculated by adding the individual scores for each component.

\section{Serum biochemical analysis}

The total biochemical indexes of serum were detected using BS-800 automatic biochemistry analyzer (Shenzhen Mindray Bio-Medical Electronics CO., LTD. China).

\section{Proteomic analysis}

The total protein in the sample was extracted, protein concentration was determined by BCA method. $10 \mu \mathrm{g}$ protein of each sample was separated by $12 \%$ SDSPAGE. The digested peptides were desalted by C18Reverse-Phase SPE Column. After being desalted, the 
peptides were separated by a C18 column $(50 \mathrm{~cm} \times$ $75 \mu \mathrm{m}$ ) on an EASY-nLCTM 1200 system (Thermo, USA) and lyophilized for mass spectrometry. All analyses were performed by a Q-Exactive HF mass spectrometer (Thermo, USA) equipped with a Nanospray Flex source (Thermo, USA).

The machine signal is transformed into peptide and protein sequence information by matching the mass spectrum output with the theoretical spectrum generated by fasta library, and then the spectrum library is established by combining the sequence information, peptide retention time and fragment ion information, so as to facilitate the subsequent DIA and PRM analysis. The original LC-MS/MS files are imported into Spectronaut Pulsar software to search and build the database. Briefly, LC-MS/MS identification were consisted of three parts. Firstly, the traditional DDA (Data-dependent Acquisition) method is used to establish the protein spectrum library. Secondly, the mass spectrum data of each sample are collected by DIA technology. At last, the mass spectrum data of candidate proteins are verified by PRM technology.

The original data of DIA is processed by Spectronaut Pulsar software. The proteins of interest to be verified were determined based on the differential protein results of DIA. Import the target proteins list into SpectroDive software (v10.1), match the DDA Library, and the software will automatically calculate the mass charge ratio of the theoretical peptide sequence of the target protein, and retain the unique peptide that meets the conditions. Export the above list and set up a mix pre scan. The pre scanned raw data was imported into SpectroDive software, and the retention time was corrected according to iRT standard peptide. Set the scheduled method and export it. Import the exported list into the inclusion list of xcalibur - PRM method editing module.

\section{Data and statistical analysis}

All experimental data obtained from rats were expressed as mean $\pm \mathrm{SD}$. A one-way repeated measure analysis of variance (ANOVA) and a log-rank test were used to determine the significance of the differences in the articular index, TSS of pathological section, biochemical analysis and PRM results, respectively.

For DIA data, difference screening conditions: foldchange $\geq 1.15$ or $\leq 0.85$ times and $p$-value $<0.05$. R package was used for the bioinformatics analysis of differentially expressed proteins, and these analyses included KOG function annotation, Go enrichment analysis, KEGG pathway classification and protein interaction analysis. Go enrichment analysis was also carried out to enrich high-level functions in three categories. Protein-protein interaction (PPI) analysis was performed using
Metascape online analysis (https://metascape.org/) and String online database (https://string-db.org/).

For PRM, quantitative results of target peptide, the quantitative information of the target peptide was derived. The quantitative value of the protein was calculated by the mean peptide quantity built in the software, and was used for statistical analysis between groups.

\begin{abstract}
Abbreviations
AP: Alternative pathway; ApoE: Apolipoprotein E; Bzw2: Basic leucine zipper and W2 domains 2; Carhsp1: Calcium-regulated heat stable protein 1; Cdc37: Cell division cycle 37; CIA: Collagen induced arthritis;

Col11a1: Collagen alpha-1(XI) chain; Col4a1: Collagen type IV alpha 1 chain; Colecl2: Collectin-12; COX: Cyclooxygenase; CP: Classical pathway;

Creb1: Cyclic AMP-responsive element-binding protein 1; Ctsd: Cathepsin D; Ctsh: Cathepsin H; DEP: Differential proteins; DIA: Data independent acquisition mass spectrometry; Eif4b: Eukaryotic initiation factor $4 \mathrm{~B}$; Elav11: Embryonic Lethal Abnormal Vision-Like Protein 1; ETD: Etodolac; Fg: Fibrinogen; Gpx1: Glutathione peroxidase; Hnrnph1: Heterogeneous nuclear ribonucleoprotein $\mathrm{H}$; HnrnpL: Heterogeneous nuclear ribonucleoprotein L; Hspa12b: Heat shock protein family A (Hsp70) member 12B; Hspa1a: Heat shock $70 \mathrm{kDa}$ protein 1A; Hspa2: Heat shock-related $70 \mathrm{kDa}$ protein 2; Hspb7: Hsp27; Klkb1: Plasma kallikrein; Lamp1: Lysosomeassociated membrane glycoprotein 1; Lum: Lumincan; Mcts1: Multiple copies in T-cell malignancy 1; MS: Mass spectrometry; Nfic: Nuclear factor I-C; NSAI Ds: Non-steroidal anti-inflammatory drugs; PG: Prostaglandin; PK: Plasma prokallikrein; PRM: Parallel reaction monitoring; Ptbp1: Polypyrimidine tractbinding proteins 1; Ptges3: Prostaglandin E synthase 3; Pura: Transcriptional activator protein Pur-a; RA: Rheumatoid arthritis; RBP: RNA binding protein; RF: Rheumatoid factor; Ruvbl1: RuvB-like 1; Septin9: Cytoskeleton interacting protein septin9; U1-70K: U1 small nuclear ribonucleoprotein 70 kDa; Snrpd3: Small nuclear ribonucleoprotein Sm D3; Srrm2: Serine/arginine repetitive matrix protein 2; Srsf7: Serine/arginine-rich splicing factor 7; Tgfbi: Transforming growth factor- $\beta$-inducible protein; Tmsb4x: Thymosin $\beta 4$; Top2a: Topoisomerase Ila; U2af2: U2 snRNP auxiliary factor large subunit; Wars: Tryptophanyl-tRNA synthetase; Yars: Tyrosyl- tRNA synthetase
\end{abstract}

\section{Supplementary Information}

The online version contains supplementary material available at https://doi. org/10.1186/s43556-021-00052-1.

Additional file 1: Supplement Fig 1. The heap maps of differentially expressed proteins.

Additional file 2: Supplement Table 1. The DDA library of the total identified proteins.

Additional file 3: Supplement Table 2. The list of total identified proteins.

\section{Acknowledgments}

We thank the Shanghai LuMing biological technology co., LTD (Shanghai, China) for providing proteomics services.

\section{Code availability}

Not applicable.

\section{Authors' contributions}

Guimin Zhang conceived the project and designed the experiments. Qin Feng, Wenkai Xia, Shenglan Wang, Guoxin Dai, Weimei Jiao, Na Guo,

Honghua Li conducted the experiments. Qin Feng performed proteomic analysis and wrote the paper. The author(s) read and approved the final manuscript.

Funding

NA 


\section{Availability of data and materials}

The data is available with the corresponding author and will be provided upon the legitimate request.

\section{Declarations}

\section{Ethics approval and consent to participate}

This study was carried out according to the National Institutes of Health Guidelines for the Care and Use of Laboratory Animals and was approved by the Animal Care and Use Committee of Shandong Province, China.

\section{Consent for publication}

All authors give consent for the publication of manuscript in Molecular Biomedicine.

\section{Competing interests}

All the authors of this paper are employees of Lunan Pharmaceutical Group, the research funds are provided by Lunan pharmaceutical Group.

\section{Author details}

${ }^{1}$ Lunan Pharmaceutical Group Co., Ltd., Linyi, China. ${ }^{2}$ Center for New Drug Safety Evaluation of Lunan Pharmaceutical, Lunan Pharmaceutical Group Co. Ltd., Linyi, China. ${ }^{3}$ National Engineering and Technology Research Center of Chirality Pharmaceutical, Lunan Pharmaceutical Group Co. Ltd., Linyi, China.

Received: 16 March 2021 Accepted: 4 August 2021

\section{Published online: 25 October 2021}

\section{References}

1. Mclnnes IB, Schett $G$. Pathogenetic insights from the treatment of rheumatoid arthritis. Lancet. 2017;389(10086):2328-37 https://doi.org/10.101 6/S0140-6736(17)31472-1.

2. Abbasi M, Mousavi MJ, Jamalzehi S, Alimohammadi R, Bezvan $\mathbf{M H}$, Mohammadi $\mathrm{H}$, et al. Strategies toward rheumatoid arthritis therapy; the old and the new. J Cell Physiol. 2019;234(7):10018-31 https://doi.org/10.1002/ jcp.27860.

3. Chen YF, Jobanputra P, Barton P, Bryan S, Fry-Smith A, Harris G, et al. Cyclooxygenase-2 selective non-steroidal anti-inflammatory drugs (etodolac, meloxicam, celecoxib, rofecoxib, etoricoxib, valdecoxib and lumiracoxib) for osteoarthritis and rheumatoid arthritis: a systematic review and economic evaluation. Health Technol Assess. 2008;12(11):1-iii https://doi.org/10.3310/ hta12110.

4. Kumagai K, Kubo M, Imai S, Toyoda F, Maeda T, Okumura N, et al. The COX2 selective blocker etodolac inhibits TNFa-induced apoptosis in isolated rabbit articular chondrocytes. Int J Mol Sci. 2013;14(10):19705-15 https://doi. org/10.3390/ijms141019705.

5. Dvornik DM. Tissue selective inhibition of prostaglandin biosynthesis by etodolac. J Rheumatol Suppl. 1997:47:40-7.

6. Tachibana M, Inoue N, Yoshida E, Matsui M, Ukai Y, Yano J. Antiinflammatory effect and low ulcerogenic activity of etodolac, a cyclooxygenase-2 selective non-steroidal anti-inflammatory drug, on adjuvant-induced arthritis in rats. Pharmacology. 2003;68(2):96-104 https:// doi.org/10.1159/000069536.

7. Zvaifler N. A review of the antiarthritic efficacy and safety of etodolac. Clin Rheumatol. 1989;8(Suppl 1):43-53 https://doi.org/10.1007/BF02214109.

8. Jones RA. Etodolac: an overview of a selective COX-2 inhibitor. Inflammopharmacology. 1999;7(3):269-75 https://doi.org/10.1007/s10787999-0010-3.

9. Hasegawa $K$, Torii $Y$, Ishii $R$, Oe $S$, Kato R, Udagawa $Y$. Effects of a selective COX-2 inhibitor in patients with uterine endometrial cancers. Arch Gynecol Obstet. 2011;284(6):1515-21 https://doi.org/10.1007/s00404-011-1883-0.

10. Ishizaki T, Katsumata K, Tsuchida A, Wada T, Mori Y, Hisada M, et al. Etodolac, a selective cyclooxygenase-2 inhibitor, inhibits liver metastasis of colorectal cancer cells via the suppression of MMP-9 activity. Int J Mol Med. 2006;17(2):357-62.

11. Nigjeh EN, Chen R, Brand RE, Petersen GM, Chari ST, von Haller PD, et al. Quantitative proteomics based on optimized data-independent Acquisition in Plasma Analysis. J Proteome Res. 2017;16(2):665-76 https://doi.org/10.1 021/acs.jproteome.6b00727.

12. Peterson AC, Russell JD, Bailey DJ, Westphall MS, Coon JJ. Parallel reaction monitoring for high resolution and high mass accuracy quantitative, targeted proteomics. Mol Cell Proteomics. 2012;11(11):1475-88 https://doi org/10.1074/mcp.0112.020131.

13. Silva ARM, Toyoshima MTK, Passarelli M, Di Mascio P, Ronsein GE. Comparing data-independent acquisition and parallel reaction monitoring in their abilities to differentiate high-density lipoprotein subclasses. J Proteome Res. 2020;19(1):248-59 https://doi.org/10.1021/acs.jproteome. 9 b00511.

14. Makinde VA, Senaldi G, Jawad AS, Berry H, Vergani D. Reflection of disease activity in rheumatoid arthritis by indices of activation of the classical complement pathway. Ann Rheum Dis. 1989;48(4):302-6 https://doi.org/1 0.1136/ard.48.4.302

15. Nandakumar KS, Holmdahl R. Antibody-induced arthritis: disease mechanisms and genes involved at the effector phase of arthritis. Arthritis Res Ther. 2006;8(6):223 https://doi.org/10.1186/ar2089.

16. Roy N, Ohtani K, Hidaka Y, Amano Y, Matsuda Y, Mori K, et al. Three pentraxins $C$-reactive protein, serum amyloid $p$ component and pentraxin 3 mediate complement activation using Collectin CL-P1. Biochim Biophys Acta Gen Subj. 2017;1861(2):1-14 https://doi.org/10.1016/j.bbagen.2016.11. 023.

17. Ma YJ, Hein E, Munthe-Fog L, Skjoedt MO, Bayarri-Olmos R, Romani L, et al. Soluble Collectin-12 (CL-12) is a pattern recognition molecule initiating complement activation via the alternative pathway. J Immunol. 2015;195(7): 3365-73 https://doi.org/10.4049/jimmunol.1500493.

18. Vogt LM, Kwasniewicz E, Talens S, Scavenius C, Bielecka E, Ekdahl KN, et al. Apolipoprotein $\mathrm{E}$ triggers complement activation in joint synovial fluid of rheumatoid arthritis patients by binding C1q. J Immunol. 2020;204(10): 2779-90 https://doi.org/10.4049/jimmunol.1900372.

19. Irmscher S, Döring N, Halder LD, Jo EAH, Kopka I, Dunker C, et al. Kallikrein cleaves C3 and activates complement. J Innate Immun. 2018;10(2):94-105 https://doi.org/10.1159/000484257.

20. Inoue K, Motonaga A, Dainaka J, Unger A, Sommerfeld L, Jansen JM, et al. Effect of etodolac on prostaglandin E2 biosynthesis, active oxygen generation and bradykinin formation. Prostaglandins Leukot Essent Fatty Acids. 1994;51(6):457-62 https://doi.org/10.1016/0952-3278(94)90065-5.

21. Björkqvist J, Jämsä A, Renné T. Plasma kallikrein: the bradykinin-producing enzyme. Thromb Haemost. 2013;110(3):399-407 https://doi.org/10.1160/ TH13-03-0258.

22. Luyendyk JP, Schoenecker JG, Flick MJ. The multifaceted role of fibrinogen in tissue injury and inflammation. Blood. 2019;133(6):511-520. https://doi. org/10.1182/blood-2018-07-818211.

23. Piersma B, Bank RA. Collagen cross-linking mediated by lysyl hydroxylase 2: an enzymatic battlefield to combat fibrosis. Essays Biochem. 2019;63(3):37787 https://doi.org/10.1042/EBC20180051.

24. Remst DF, Blaney Davidson EN, van der Kraan PM. Unravelling osteoarthritisrelated synovial fibrosis: a step closer to solving joint stiffness. Rheumatology (Oxford). 2015;54(11):1954-63 https://doi.org/10.1093/ rheumatology/kev228.

25. Goldstein AL, Hannappel E, Sosne G, Kleinman HK. Thymosin B4: a multifunctional regenerative peptide. Basic properties and clinical applications. Expert Opin Biol Ther. 2012;12(1):37-51 https://doi.org/10.1517/14712598.2 012.634793

26. Chu Y, You M, Zhang J, Gao G, Han R, Luo W, et al. Adipose-derived mesenchymal stem cells enhance ovarian Cancer growth and metastasis by increasing Thymosin Beta 4X-linked expression. Stem Cells Int. 2019;2019: 9037197. https://doi.org/10.1155/2019/9037197-9.

27. Franklin S, Vondriska TM. Genomes, proteomes, and the central dogma. Circ Cardiovasc Genet. 2011;4(5):576 https://doi.org/10.1161/CIRCGENETICS.110. 957795.

28. Zhao C, Hui W, Fernandes MJ, Poubelle PE, Bourgoin SG. Lysophosphatidic acid-induced IL-8 secretion involves MSK1 and MSK2 mediated activation of CREB1 in human fibroblast-like synoviocytes. Biochem Pharmacol. 2014; 90(1):62-72 https://doi.org/10.1016/j.bcp.2014.04.012.

29. Li Y, Fu Y, Hu X, Sun L, Tang D, Li N, et al. The HBx-CTTN interaction promotes cell proliferation and migration of hepatocellular carcinoma via CREB1. Cell Death Dis. 2019;10(6):405 https://doi.org/10.1038/s41419-019-1 650-x.

30. Kaminski R, Darbinyan A, Merabova N, Deshmane SL, White MK, Khalili K. Protective role of Puralpha to cisplatin. Cancer Biol Ther. 2008;7(12):1926-35 https://doi.org/10.4161/cbt.7.12.6938.

31. Xu G, Zhang Y, Li N, Wu Y, Zhang J, Xu R, et al. LBX2-AS1 up-regulated by $\mathrm{NFIC}$ boosts cell proliferation, migration and invasion in gastric cancer 
through targeting miR-491-5p/ZNF703. Cancer Cell Int. 2020;20(1):136. https://doi.org/10.1186/s12935-020-01207-w.

32. Zhang J, Jiang HY, Zhang LK, Xu WL, Qiao YT, Zhu XG, et al. C-FLIPL modulated Wnt/B-catenin activation via association with TIP49 protein. J Biol Chem. 2017;292(6):2132-42 https://doi.org/10.1074/jbc.M1 16.753251.

33. Corsiero E, Bombardieri M, Carlotti E, Pratesi F, Robinson W, Migliorini P, et al. Single cell cloning and recombinant monoclonal antibodies generation from RA synovial B cells reveal frequent targeting of citrullinated histones of NETs. Ann Rheum Dis. 2016;75(10):1866-75 https://doi.org/1 0.1136/annrheumdis-2015-208356.

34. Jones PA, Issa JP, Baylin S. Targeting the cancer epigenome for therapy. Nat Rev Genet. 2016;17(10):630-41 https://doi.org/10.1038/nrg.2016.93.

35. Zuklys KL, Szer IS, Szer W. Autoantibodies to DNA topoisomerase II in juvenile rheumatoid arthritis. Clin Exp Immunol. 1991;84(2):245-9 https://doi. org/10.1111/j.1365-2249.1991.tb08156.x.

36. Chen T, Sun Y, Ji P, Kopetz S, Zhang W. Topoisomerase lla in chromosome instability and personalized cancer therapy. Oncogene. 2015;34(31):4019-31 https://doi.org/10.1038/onc.2014.332.

37. Siebring-van Olst E, Blijlevens M, de Menezes RX, van der Meulen-Muileman ${ }_{H}$, Smit EF, van Beusechem WW. A genome-wide siRNA screen for regulators of tumor suppressor p53 activity in human non-small cell lung cancer cells identifies components of the RNA splicing machinery as targets for anticancer treatment. Mol Oncol. 2017;11(5):534-51 https://doi.org/10.1 002/1878-0261.12052.

38. Greidinger EL, Foecking MF, Magee J, Wilson L, Ranatunga S, Ortmann RA, et al. A major B cell epitope present on the apoptotic but not the intact form of the U1-70-kDa ribonucleoprotein autoantigen. J Immunol. 2004; 172(1):709-16 https://doi.org/10.4049/jimmunol.172.1.709.

39. Pierceall WE, Wolfe M, Suschak J, Chang H, Chen Y, Sprott KM, et al. Strategies for $\mathrm{H}$-score normalization of preanalytical technical variables with potential utility to immunohistochemical-based biomarker quantitation in therapeutic response diagnostics. Anal Cell Pathol (Amst). 2011;34(3):159-68 https://doi.org/10.3233/ACP-2011-014.

40. Li J, Cheng D, Zhu M, Yu H, Pan Z, Liu L, et al. OTUB2 stabilizes U2AF2 to promote the Warburg effect and tumorigenesis via the AKT/mTOR signaling pathway in non-small cell lung cancer. Theranostics. 2019;9(1):179-95 https://doi.org/10.7150/thno.29545.

41. Chen S, Lv L, Zhan Z, Wang X, You Z, Luo X, et al. Silencing of long noncoding RNA SRRM2-AS exerts suppressive effects on angiogenesis in nasopharyngeal carcinoma via activating MYLK-mediated cGMP-PKG signaling pathway. J Cell Physiol. 2020;235(11):7757-68 https://doi.org/10.1 002/jcp.29382

42. Fu Y, Wang Y. SRSF7 knockdown promotes apoptosis of colon and lung cancer cells. Oncol Lett. 2018;15(4):5545-52 https://doi.org/10.3892/ol.2018. 8072.

43. Abdelmohsen K, Gorospe M. Posttranscriptional regulation of cancer traits by HuR. Wiley Interdiscip Rev RNA. 2010;1(2):214-29 https://doi.org/10.1002/ wrna.4.

44. Monzón-Casanova E, Matheson LS, Tabbada K, Zarnack K, Smith CW, Turner M. Polypyrimidine tract-binding proteins are essential for B cell development. Elife. 2020;9:e53557 https://doi.org/10.7554/eLife.53557.

45. Monzón-Casanova E, Screen M, Díaz-Muñoz MD, Coulson RMR, Bell SE, Lamers $\mathrm{G}$, et al. The RNA-binding protein PTBP1 is necessary for $\mathrm{B}$ cell selection in germinal centers. Nat Immunol. 2018;19(3):267-78 https://doi. org/10.1038/s41590-017-0035-5

46. Georgilis A, Klotz S, Hanley CJ, Herranz N, Weirich B, Morancho B, et al. PTBP1-Mediated Alternative Splicing Regulates the Inflammatory Secretome and the Pro-tumorigenic Effects of Senescent Cells. Cancer Cell. 2018;34(1): 85-102.e9. https://doi.org/10.1016/j.ccell.2018.06.007.

47. Pararajalingam P, Coyle KM, Arthur SE, Thomas N, Alcaide M, Meissner B, et al. Coding and noncoding drivers of mantle cell lymphoma identified through exome and genome sequencing. Blood. 2020;136(5):572-84 https://doi.org/10.1182/blood.2019002385.

48. Li Z, Chao TC, Chang KY, Lin N, Patil VS, Shimizu C, et al. The long noncoding RNA THRIL regulates TNFa expression through its interaction with hnRNPL. Proc Natl Acad Sci U S A. 2014;111(3):1002-7 https://doi.org/1 0.1073/pnas. 1313768111.

49. Fei $T$, Chen $Y$, Xiao $T$, Fei $T$, Chen $Y$, Xiao $T$, et al. Genome-wide CRISPR screen identifies HNRNPL as a prostate cancer dependency regulating RNA splicing. Proc Natl Acad Sci U S A. 2017;114(26):E5207-15 https://doi.org/1 0.1073/pnas. 1617467114
50. Yang PP, Yu XH, Zhou J. Tryptophanyl-tRNA synthetase (WARS) expression in uveal melanoma - possible contributor during uveal melanoma progression. Biosci Biotechnol Biochem. 2020;84(3):471-80 https://doi.org/1 0.1080/09168451.2019.1686967.

51. Zhang $C$, Lin $X$, Zhao $Q$, Wang $Y$, Jiang F, Ji C, et al. YARS as an oncogenic protein that promotes gastric cancer progression through activating PI3KAkt signaling. J Cancer Res Clin Oncol. 2020;146(2):329-42 https://doi.org/1 0.1007/s00432-019-03115-7.

52. Huang L, Chen S, Fan H, Ai F, Sheng W. BZW2 promotes the malignant progression of colorectal cancer via activating the ERK/MAPK pathway. J Cell Physiol. 2020;235(5):4834-42 https://doi.org/10.1002/jcp.29361.

53. Chang G, Zheng J, Xiao W, Chang S, Wei Q, Wu H, et al. PKC inhibition of sotrastaurin has antitumor activity in diffuse large B-cell lymphoma via regulating the expression of MCT-1. Acta Biochim Biophys Sin Shanghai. 2018;50(4):399-407 https://doi.org/10.1093/abbs/gmy021.

54. Hernández G, Vázquez-Pianzola P, Zurbriggen A, Altmann M, Sierra JM, Rivera-Pomar R. Two functionally redundant isoforms of Drosophila melanogaster eukaryotic initiation factor $4 \mathrm{~B}$ are involved in cap-dependent translation, cell survival, and proliferation. Eur J Biochem. 2004;271(14):292336 https://doi.org/10.1111/j.1432-1033.2004.04217.x.

55. Mi B, Liu G, Zhou W, Lv H, Liu Y, Liu J. Identification of genes and pathways in the synovia of women with osteoarthritis by bioinformatics analysis. Mol Med Rep. 2018;17(3):4467-73 https://doi.org/10.3892/mmr.2018.8429.

56. Catez F, Dalla Venezia N, Marcel V, Zorbas C, Lafontaine DLJ, Diaz JJ. Ribosome biogenesis: an emerging druggable pathway for cancer therapeutics. Biochem Pharmacol. 2019;159:74-81 https://doi.org/10.1016/j. bcp.2018.11.014.

57. Hou H, Wang F, Zhang W, Wang D, Li X, Bartlam M, et al. Structurefunctional analyses of CRHSP-24 plasticity and dynamics in oxidative stress response. J Biol Chem. 2011;286(11):9623-35 https://doi.org/10.1074/jbc.M11 0.177436 .

58. Tiwari R, Sahu I, Soni BL, Sathe GJ, Datta KK, Thapa P, et al. Quantitative phosphoproteomic analysis reveals system-wide signaling pathways regulated by site-specific phosphorylation of Keratin-8 in skin squamous cell carcinoma derived cell line. Proteomics. 2017;17(7):https://doi.org/10.1002/ pmic.201600254.

59. Katano M, Okamoto K, Arito M, Kawakami Y, Kurokawa MS, Suematsu N, et al. Implication of granulocyte-macrophage colony-stimulating factor induced neutrophil gelatinase-associated lipocalin in pathogenesis of rheumatoid arthritis revealed by proteome analysis. Arthritis Res Ther. 2009; 11(1):R3 https://doi.org/10.1186/ar2587.

60. Ashraf $Y$, Mansouri $H$, Laurent-Matha V, Alcaraz LB, Roger P, Guiu S, et al. Immunotherapy of triple-negative breast cancer with cathepsin D-targeting antibodies. J Immunother Cancer. 2019;7(1):29 https://doi.org/10.1186/s4042 5-019-0498-Z.

61. Jevnikar Z, Rojnik M, Jamnik P, Doljak B, Fonovic UP, Kos J. Cathepsin H mediates the processing of Talin and regulates migration of prostate cancer cells. J Biol Chem. 2013;288(4):2201-9 https://doi.org/10.1074/jbc.M112.4363 94.

62. Alessandrini F, Pezzè L, Ciribilli Y. LAMPs: shedding light on cancer biology. Semin Oncol. 2017;44(4):239-53 https://doi.org/10.1053/.jseminoncol.2017.1 0.013

63. Miettinen JJ, Kumari R, Traustadottir GA, Huppunen ME, Sergeev $P$, Majumder MM, et al. Aminopeptidase expression in multiple myeloma associates with disease progression and sensitivity to Melflufen. Cancers (Basel). 2021;13(7):1527 https://doi.org/10.3390/cancers13071527.

64. Verbrugge SE, Scheper RJ, Lems WF, de Gruil TD, Jansen G. Proteasome inhibitors as experimental therapeutics of autoimmune diseases. Arthritis Res Ther. 2015;17(1):17 https://doi.org/10.1186/s13075-015-0529-1.

65. Grigoreva TA, Tribulovich VG, Garabadzhiu AV, Melino G, Barlev NA. The 265 proteasome is a multifaceted target for anti-cancer therapies. Oncotarget. 2015;6(28):24733-49 https://doi.org/10.18632/oncotarget.4619.

66. Chorazy PA, Schumacher HR Jr, Edlind TD. Role of glutathione peroxidase in rheumatoid arthritis: analysis of enzyme activity and DNA polymorphism. DNA Cell Biol. 1992;11(3):221-5 https://doi.org/10.1089/dna.1992.11.221.

67. Wei R, Qiu H, Xu J, Mo J, Liu Y, Gui Y, et al. Expression and prognostic potential of GPX1 in human cancers based on data mining. Ann Transl Med. 2020;8(4):124 https://doi.org/10.21037/atm.2020.02.36

68. Tukaj S, Mantej J, Sobala M, Potrykus K, Sitko K. Autologous extracellular Hsp70 exerts a dual role in rheumatoid arthritis. Cell Stress Chaperones. 2020;25(6):1105-10 https://doi.org/10.1007/s12192-020-01114-z. 
69. Kumar S, Stokes J 3rd, Singh UP, Scissum Gunn K, Acharya A, Manne U, et al. Targeting Hsp70: a possible therapy for cancer. Cancer Lett. 2016; 374(1):156-66 https://doi.org/10.1016/j.canlet.2016.01.056.

70. Sedlackova L, Sosna A, Vavrincova P, Frýdl J, Guerriero V, Raynes DA, et al. Heat shock protein gene expression profile may differentiate between rheumatoid arthritis, osteoarthritis, and healthy controls. Scand J Rheumatol 2011;40(5):354-7 https://doi.org/10.3109/03009742.2011.552522.

71. Acunzo J, Andrieu C, Baylot V, So A, Rocchi P. Hsp27 as a therapeutic target in cancers. Curr Drug Targets. 2014;15(4):423-31 https://doi.org/10.2174/13 894501113146660230.

72. Bharadwaj S, Lee KE, Dwivedi VD, Yadava U, Kang SG. Computational aided mechanistic understanding of Camellia sinensis bioactive compounds against co-chaperone p23 as potential anticancer agent. J Cell Biochem. 2019;120(11):19064-75 https://doi.org/10.1002/jcb.29229.

73. Zhu J, Yan F, Tao J, Zhu X, Liu J, Deng S, et al. Cdc37 facilitates cell surviva of colorectal carcinoma via activating the CDK4 signaling pathway. Cancer Sci. 2018;109(3):656-65 https://doi.org/10.1111/cas.13495.

74. Connolly D, Hoang HG, Adler E, Tazearslan C, Simmons N, Bernard W, et al. Septin 9 amplification and isoform-specific expression in peritumoral and tumor breast tissue. Biol Chem. 2014;395(2):157-67 https://doi.org/10.1515/ hsz-2013-0247.

75. Marcus J, Bejerano-Sagie M, Patterson N. Agchi S, Verkhusha W, Connolly D, et al. Septin 9 isoforms promote tumorigenesis in mammary epithelial cells by increasing migration and ECM degradation through metalloproteinase secretion at focal adhesions. Oncogene. 2019;38(30):5839-59 https://doi. org/10.1038/s41388-019-0844-0.

76. Veys EM. Clinical performance of etodolac in patients with osteoarthritis and rheumatoid arthritis. Eur J Rheumatol Inflamm. 1994;14(1):23-7.

77. Lightfoot R. Comparison of the efficacy and safety of etodolac and piroxicam in patients with rheumatoid arthritis. Etodolac study 326 rheumatoid arthritis investigators group. J Rheumatol Suppl. 1997;47:10-6.

78. Holers VM, Banda NK. Complement in the initiation and evolution of rheumatoid arthritis. Front Immunol. 2018;9:1057 https://doi.org/10.3389/ fimmu.2018.01057.

79. Smolen JS, Aletaha D, McInnes IB. Rheumatoid arthritis [published correction appears in lancet. 2016 Oct 22;388(10055):1984]. Lancet. 2016; 388(10055):2023-38 https://doi.org/10.1016/S0140-6736(16)30173-8.

80. Hoppe B, Dörner T. Coagulation and the fibrin network in rheumatic disease: a role beyond haemostasis. Nat Rev Rheumatol. 2012;8(12):738-46 https://doi.org/10.1038/nrrheum.2012.184.

81. Craciun FL, Ajay AK, Hoffmann D, Saikumar J, Fabian SL, Bijol V, et al. Pharmacological and genetic depletion of fibrinogen protects from kidney fibrosis. Am J Physiol Renal Physiol. 2014;307(4):F471-84 https://doi.org/1 0.1152/ajprenal.00189.2014

82. Wang Y, Sun Y, Feng J, Li Z, Yu H, Ding X, et al. Glycopatterns and glycoproteins changes in MCN and SCN: a prospective cohort study. Biomed Res Int. 2019;2019:2871289. https://doi.org/10.1155/2019/287128911.

83. Chen $\mathrm{YH}$, Keegan S, Kahli M, Tonzi P, Fenyö D, Huang TT, et al. Transcription shapes DNA replication initiation and termination in human cells. Nat Struct Mol Biol. 2019;26(1):67-77 https://doi.org/10.1038/s41594-018-0171-0.

84. Wang GS, Cooper TA. Splicing in disease: disruption of the splicing code and the decoding machinery. Nat Rev Genet. 2007;8(10):749-61 https://doi. org/10.1038/nrg2164.

85. Díaz-Muñoz MD, Turner M. Uncovering the role of RNA-binding proteins in gene expression in the immune system. Front Immunol. 2018;9:1094 https://doi.org/10.3389/fimmu.2018.01094.

86. Lee SC, Abdel-Wahab O. Therapeutic targeting of splicing in cancer. Nat Med. 2016;22(9):976-86 https://doi.org/10.1038/nm.4165.

87. Bonam SR, Wang F, Muller S. Lysosomes as a therapeutic target. Nat Rev Drug Discov. 2019;18(12):923-48 https://doi.org/10.1038/s41573-019-0036-1.

88. Sohar N, Hammer H, Sohar I. Lysosomal peptidases and glycosidases in rheumatoid arthritis. Biol Chem. 2002;383(5):865-9 https://doi.org/10.1515/ BC.2002.093.

89. Siklos M, BenAissa M, Thatcher GR. Cysteine proteases as therapeutic targets: does selectivity matter? A systematic review of calpain and cathepsin inhibitors. Acta Pharm Sin B. 2015;5(6):506-19 https://doi.org/10.1016/j.a psb.2015.08.001.

90. Jackson G, Einsele H, Moreau P, Miguel JS. Bortezomib, a novel proteasome inhibitor, in the treatment of hematologic malignancies. Cancer Treat Rev. 2005;31(8):591-602 https://doi.org/10.1016/j.ctrv.2005.10.001.
91. Wang L, Zhang L, Li L, Zheng Z, Shang J, Wang C, et al. Small-molecule inhibitor targeting the Hsp90-Cdc37 protein-protein interaction in colorectal cancer. Sci Adv. 2019;5(9):eaax2277. https://doi.org/10.1126/sciadv.aax2277

92. Ciocca DR, Calderwood SK. Heat shock proteins in cancer: diagnostic, prognostic, predictive, and treatment implications. Cell Stress Chaperones. 2005;10(2):86-103 https://doi.org/10.1379/csc-99r.1.

93. Wu J, Liu T, Rios Z, Mei Q, Lin X, Cao S. Heat shock proteins and Cancer. Trends Pharmacol Sci. 2017;38(3):226-56 https://doi.org/10.1016/j.tips.201 6.11 .009 .

94. Bustamante MF, Garcia-Carbonell R, Whisenant KD, Guma M. Fibroblast-like synoviocyte metabolism in the pathogenesis of rheumatoid arthritis. Arthritis Res Ther. 2017;19(1):110 https://doi.org/10.1186/s13075-017-1303-3.

95. Olumuyiwa-Akeredolu OO, Pretorius E. Rheumatoid arthritis: notable biomarkers linking to chronic systemic conditions and Cancer. Curr Pharm Des. 2016;22(7):918-24 https://doi.org/10.2174/138161282266615120915353 5.

96. Klein A, Polliack A, Gafter-Gvili A. Rheumatoid arthritis and lymphoma: incidence, pathogenesis, biology, and outcome. Hematol Oncol. 2018;36(5): 733-9 https://doi.org/10.1002/hon.2525.

97. Tseng HW, Lu LY, Lam HC, Tsai KW, Huang WC, Shiue YL. The influence of disease-modifying anti-rheumatic drugs and corticosteroids on the association between rheumatoid arthritis and skin cancer: a nationwide retrospective case-control study in Taiwan. Clin Exp Rheumatol. 2018;36(3): 471-8.

98. Morand S, Staats H, Creeden JF, lqbal A, Kahaleh B, Stanbery L, et al. Molecular mechanisms underlying rheumatoid arthritis and cancer development and treatment. Future Oncol. 2020;16(9):483-95 https://doi. org/10.2217/fon-2019-0722.

99. Krenn V, Morawietz L, Häupl T, Neidel J, Petersen I, König A. Grading of chronic synovitis--a histopathological grading system for molecular and diagnostic pathology. Pathol Res Pract. 2002;198(5):317-25 https://doi.org/1 0.1078/0344-0338-5710261.

\section{Publisher's Note}

Springer Nature remains neutral with regard to jurisdictional claims in published maps and institutional affiliations. 